
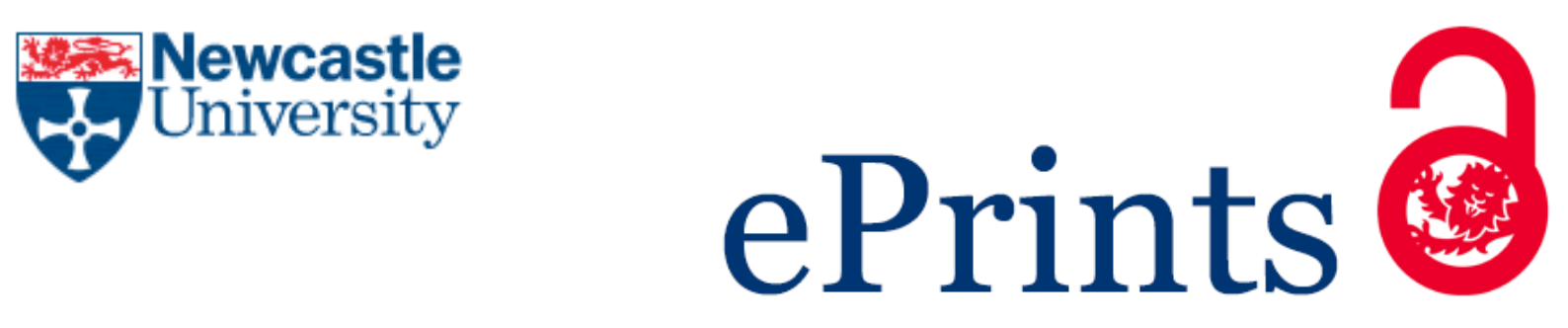

Vannella KM, Ramalingam TR, Borthwick LA, Barron L, Hart KM, Thompson RW, Kindrachuk KN, Cheever AW, White S, Budelsky AL, Comeau MR, Smith DE, Wynn TA.

Combinatorial targeting of TSLP, IL-25, and IL-33 in type 2 cytokine-driven inflammation and fibrosis.

Science Translational Medicine 2016, 8(337).

\title{
Copyright:
}

This is the author's version of the work. It is posted here by permission of the AAAS for personal use, not for redistribution. The definitive version was published in Science Translational Medicine on 8(337) 04/05/2016, http://dx.doi.org/10.1126/scitranslmed.aaf1938

DOI link to article:

http://dx.doi.org/10.1126/scitranslmed.aaf1938

Date deposited:

$01 / 07 / 2016$ 
Title:

*Dr. Thomas A. Wynn, Chief: Immunopathogenesis Section, Laboratory of Parasitic Diseases,

\section{Combinatorial Targeting of TSLP, IL-25, and IL-33 in Type 2 Cytokine-driven} Inflammation and Fibrosis
Authors: Kevin M. Vannella ${ }^{1}$, Thirumalai R. Ramalingam ${ }^{1}$, Lee A. Borthwick ${ }^{1,2}$, Luke Barron ${ }^{1}$, Kevin M. Hart ${ }^{1}$, Robert W. Thompson ${ }^{1}$, Kristen N. Kindrachuk ${ }^{1}$, Allen W. Cheever ${ }^{1,3}$, Sandra White $^{1}$, Alison L. Budelsky ${ }^{4}$, Michael R. Comeau ${ }^{4}$, Dirk E. Smith ${ }^{4}$, and Thomas A.Wynn ${ }^{1 *}$

\section{Affiliations:}

${ }^{1}$ Program in Tissue Immunity and Repair, Laboratory of Parasitic Diseases, National Institute of Allergy and Infectious Diseases, National Institutes of Health; Bethesda, MD 20892; USA;

${ }^{2}$ Tissue Fibrosis and Repair Group, Institute of Cellular Medicine, Newcastle University;

Newcastle upon Tyne, NE2 4HH; UK;

${ }^{3}$ Biomedical Research Institute; Rockville, MD 20852; USA;

${ }^{4}$ Department of Inflammation Research, Amgen, 1201 Amgen Court West, Seattle, WA 98119, USA 
Memorial Dr, Rm. 211C, Bethesda, MD 20892, Telephone: 301-496-4758, E-mail: twynn@niaid.nih.gov, Fax: 301-480-5025

One Sentence Summary: Although TSLP, IL-25, and IL-33 have emerged as important initiators of type 2 immunity, combined blockade of all three mediators may be needed to treat some forms of progressive type 2 cytokine driven inflammation and fibrosis.

Abstract: Thymic stromal lymphopoietin (TSLP), IL-25, and IL-33 are important initiators of type 2-associated mucosal inflammation and immunity. However, their role in the maintenance of progressive type 2 inflammation and fibrosis is much less clear. Here, using chronic models of helminth infection and allergic lung inflammation, we show that collective disruption of TSLP, IL-25, and IL-33 signaling suppresses chronic and progressive type 2 cytokine-driven inflammation and fibrosis. In a schistosome lung granuloma model or during chronic S. mansoni infection in the liver, individual ablation of TSLP, IL-25, or IL-33/ST2 had no impact on the development of IL-4/IL-13-dependent inflammation or fibrosis. However, significant reductions in granuloma-associated eosinophils, hepatic fibrosis, and IL-13-producing group 2 innate lymphoid cells (ILC2s) were observed when signaling of all three mediators was simultaneously disrupted. Combined blockade via mAb treatment also reduced IL-5 and IL-13 expression during primary and secondary granuloma formation in the lung. In a model of chronic house dust mite-induced allergic lung inflammation, combined mAb treatment did not decrease established inflammation or fibrosis. TSLP/IL-33 double-knockout mice treated with anti-IL-25 mAb during priming, however, displayed decreased inflammation, mucus production, and lung remodeling in the chronic phase. Together, these studies reveal partially redundant roles for TSLP, IL-25, and 
IL-33 in the maintenance of type 2 pathology and suggest that in some settings, early combined targeting of these mediators is necessary to ameliorate progressive type 2-driven disease.

Main Text:

\section{Introduction}

Type 2 immunity is characterized by the production of the cytokines IL-4, IL-5, IL-9, and IL-13, which play diverse roles in the immune response (1). In addition to suppressing the proinflammatory activity of type 1 immune responses (2), type 2 immunity regulates wound healing (3), metabolic homeostasis (4), and immunity to several extracellular parasites (5). However, while the type 2 response exhibits many host protective functions, should these responses persist or become dysregulated, they can contribute to the development of disease. Chronic type 2 cytokine production underlies diseases including allergic asthma, atopic dermatitis, allergic rhinitis, ulcerative colitis, and many chronic fibroproliferative disorders (6-9). Therefore, a better understanding of the mechanisms that regulate the initiation, maintenance and resolution of type 2 immune responses could reveal novel approaches to treat a host of important human diseases.

Three predominantly epithelial cell-derived cytokines: thymic stromal lymphopoietin (TSLP), IL-25, and IL-33, have emerged as important initiators of type 2 immunity in mammals, and their expression during type 2 disease in humans is widely-documented (10-15). These alarmins are released from the epithelium and other local stromal compartments when cells are damaged or stressed by allergens, pollutants or pathogens and thereby trigger the production of 
the canonical type 2 cytokines IL-5, IL-9, and IL-13 by human and mouse cells of the innate and adaptive immune system $(16,17)$. TSLP targets dendritic cells (DCs), basophils, mast cells, monocytes, natural killer T cells, and type 2 innate lymphoid cells (ILC2s) (18-21). In humans, TSLP has been shown to induce naïve human $\mathrm{CD} 4^{+} \mathrm{Th} 2$ cell responses, but only in the presence of DCs (22). IL-25 and IL-33 exhibit similar Th2-inducing activity, but rather than targeting DCs, myeloid cells, and Th2 cells, they largely promote type 2 immunity by stimulating ILC2s as well as basophils, mast cells, and eosinophils. IL-33 will amplify antigen-dependent and independent effector responses from both human and mouse Th2 cells $(16,17)$. One recent study revealed that IL-33 can enhance TSLP and DC-mediated human Th2 memory responses in vitro suggesting the alarmins could play a role in maintaining immune responses (23). Although TSLP, IL-25, and IL-33 have all been shown to promote type 2 immunity when overexpressed in mice (10-12), the requirement for these cytokines in the development of type 2 immunity in response to allergens and helminth parasites has been more variable, with some studies identifying little to no role for TSLP, IL-25, or IL-33 when targeted individually (24-28). This variability has been attributed to the redundant and overlapping functional activities of these cytokines. IL-33 and IL-25 have both been shown to induce production of IL-13 by human ILCs in vitro, for example (29). However, this theory has not been systematically investigated in vivo, nor have the combined roles of the 3 cytokines been dissected in models of chronic type 2dependent disease.

In the present study, we utilized both genetic- and monoclonal antibody-based strategies to investigate whether bi-functional or tri-functional targeting of TSLP, IL-25, and IL-33dependent signaling more effectively controls pathogenic Th2 responses than disrupting any of the pathways individually. The roles of the 3 cytokines in the initiation and maintenance of 
primary and secondary type 2 immune responses were investigated in both acute and chronic models of lung inflammation and during chronic helminth infection. These models involve innate-initiated pathways as well as the development of antigen-specific $\mathrm{T}$ cell responses that influence outcomes at later stages. A major goal was to investigate if type 2 cytokine-driven inflammation and fibrosis could be ameliorated more effectively if all three epithelial cytokines were targeted in combination. Moreover, in contrast to previous studies that have focused on their role in the "initiation" of type 2 immunity (30), our studies were also designed to investigate if TSLP, IL-25, and IL-33, either alone or in combination, are required for the "maintenance" of established type 2-driven disease, as this is the stage where most therapeutic strategies are initiated.

\section{Results}

\section{Function of IL-25 during the initiation and maintenance of type 2 inflammation}

We have previously shown that TSLP is not required for type 2-driven granuloma formation and fibrosis induced by the eggs of the helminth parasite Schistosoma mansoni (26). Another group has demonstrated that many helminths could bypass the need for TSLP in the development of type 2 responses by directly modulating dendritic cell function (28). However, the relative importance of IL-25 and IL-33 to the maintenance of established type 2-driven disease and the potential redundancy of these mediators has not been assessed. Therefore, we began by exploring the contribution of IL-25 in type 2-dependent inflammation and fibrosis by overexpressing IL-25 in mice that were injected i.v. with live $S$. mansoni eggs. Hydrodynamic delivery of an IL-25-expressing plasmid to naïve mice boosted IL-25 mRNA expression more 
than 1000-fold in the liver (Fig. 1A). As observed in previous studies (10), corresponding increases in IL-4, IL-5, and IL-13 expression were observed in both the liver and the lung (Fig.

118 1A). When the IL-25-expressing plasmid was delivered 24 hours prior to exposure to $S$. mansoni 119 eggs, the resulting granulomatous response to the eggs in the lung was exacerbated (Fig. 1B). 120 Indeed, granuloma volume more than doubled in the IL-25 pre-treated mice, and their lesions 121 contained many more eosinophils than control mice, which was likely due to type 2 cytokine 122 induction in the lung (Fig. 1C). Goblet cell hyperplasia and mucus production were also 123 augmented in lungs of mice treated with the IL-25 plasmid. The effects of IL-25 plasmid 124 administration were reduced in IL-13R $\alpha^{-/-}$mice, demonstrating that the IL-25-mediated 125 increase in type 2-associated pathology was dependent on IL-4/IL-13-mediated signaling through 126 the type II IL-4 receptor complex (Fig. 1B). Eosinophils accumulated following plasmid 127 administration, however, likely explained by IL-25-driven IL-5 expression (Fig. 1C). Although these studies established that IL-25 could exacerbate type 2 cytokine-driven 129 pathology, they did not reveal whether endogenously expressed IL-25 was critical to the 130 development of granulomatous inflammation and fibrosis. To clarify the role of IL-25 in both 131 the initiation and maintenance of type 2-driven fibrosis, we used IL-25/- mice in both primary 132 (like Fig. 1B) and secondary i.v. S. mansoni egg challenge models (31). In these experiments, 133 naïve or egg-sensitized IL-25/- mice and wild-type littermates were challenged i.v. with live $S$. 134 mansoni eggs and granuloma formation was quantified on day 7 post-challenge. Neither primary 135 nor secondary granuloma formation was significantly reduced in the absence of IL-25 (Fig. 1D). 136 The number of granulomatous eosinophils in each group was also indistinguishable during both 137 primary and secondary challenges (Fig. 1D, right panel). Finally, to evaluate the requirement 138 for IL-25 in a more chronic type 2 disease setting, we exposed wild-type and IL-25/- mice to $S$. 
mansoni cercariae and quantified granuloma volume, tissue eosinophilia, and fibrosis in the liver after 12 weeks of infection. Similar to the results in the lung (Fig. 1D), no significant change in type 2-dependent pathology was observed in livers of IL-25/-- mice compared with wild-type mice when chronically infected with $S$. mansoni (Fig. 1E).

\section{Role of IL-33 in type 2 inflammation and fibrosis}

Given that the inflammation and fibrosis induced by S. mansoni eggs in both the lung and liver were IL-4-, IL-13-, and IL-13R $\alpha$-dependent but did not require IL-25 or TSLP (26), we next examined whether IL-33/ST2 receptor signaling was required in this setting. As observed in IL$25^{-/-}$mice, mice deficient in IL-33 showed no significant reduction in either primary (Fig. 2A) or secondary granuloma formation (Fig. 2B) when challenged i.v. with live S. mansoni eggs. In both models, type 2-driven fibrosis and eosinophilia were similar in wild-type and IL-33-- mice. We also infected wild-type and IL-33 $3^{-/}$mice with $S$. mansoni cercariae and examined the development of type 2-dependent pathology in the liver at acute (week 9) and chronic (week 12) phases of infection. Although recent studies using hepatotoxic chemicals or schistosome eggdriven models have suggested that IL-33 expression is critical to the development of fibrosis in the liver (32), we observed no reduction in hepatic fibrosis in IL-33 $3^{-/}$mice at either time-point (Fig. 2C) and picrosirius red staining of liver sections (Fig. 2D). The number of eosinophils in the lesions and the diameter of granulomas were also similar in the absence of IL-33, confirming unimpaired type 2-driven inflammation (Fig. 2E). The marked type 2 cytokine response that normally develops in the livers of infected wild-type mice was also similarly observed in IL-33 ${ }^{-/-}$ mice, and in the case of IL-4 expression was even slightly increased (Fig. 2F), further suggesting 
that IL-33 signaling is dispensable for the development of type 2 cytokine-driven pathology during both acute and chronic S. mansoni infection.

\section{Disrupting TSLP, IL-25, and IL-33 signaling during S. mansoni infection}

To investigate whether TSLP, IL-25, and IL-33 were playing redundant roles in the maintenance of type 2 cytokine-dependent granuloma formation and fibrosis, we developed strategies to disrupt all three cytokine pathways simultaneously. In initial studies, $\mathrm{TSLP}^{-/-}$mice were crossed with IL-33 ${ }^{-/}$mice to generate a double knockout mouse, and a highly effective neutralizing mAb was introduced to the double knockout mice to block IL-25. C57BL/6 mice were infected with S. mansoni cercariae, and the response in the absence of TSLP, IL-25, and IL-33 signaling was evaluated at acute (week 9) and chronic (week 12) phases of infection. We first measured TSLP, IL-25, and IL-33 gene expression and found each gene is constitutively expressed in whole liver tissue at detectable levels (Fig. S1). These levels of expression do not change significantly during S. mansoni infection on a whole tissue level. In contrast to the studies in which individual cytokines were targeted, we observed a small yet significant decrease in granuloma volume in the triple deficient mice in the acute phase (Fig. 3A). This was also accompanied by a $25-30 \%$ decrease in hepatic fibrosis (Fig. 3B) and a small yet significant decrease in the number of granuloma-associated eosinophils (Fig. 3C). Interestingly, the decrease in pathology observed at week 9 was associated with a significant decrease in the frequency of IL-13-producing type 2 innate lymphoid cells (ILC2s) in the mesenteric lymph nodes (MLNs) (Fig. 3D), which is consistent with the ILC2-promoting activity of IL-25 and IL-33 (33). The frequency of ILC2s in the liver, however, was not significantly different between the two groups (Fig. 3D, right panel). Total leukocyte numbers were similar in the liver tissue and MLNs of both cohorts. 

week 9 was no longer significant (Fig. 3D, left panel), and while there was a modest but consistent decrease in pathology at week 9, granuloma volume and fibrosis became indistinguishable between WT and DKO $+\alpha$ IL-25-treated mice (Figs. 3A-C). Indeed, both groups of mice displayed a striking increase in IL-13-dependent fibrosis by week 12 as determined by both hydroxyproline assay (Fig. 3B) and picrosirius red staining (Fig. 3E). In addition, while the frequency of IL-13-producing ILC2s was lower in the MLN at week 9 (Fig. 3D), a marked increase in IL-4- and IL-13-producing CD4 ${ }^{+} \mathrm{T}$ cells was observed at the same time point in the granulomatous livers of the DKO $+\alpha$ IL-25-treated mice (Fig. 3F). Antigenspecific $\mathrm{CD}^{+} \mathrm{Th} 2$ cell cytokine production likely compensated for the transient decrease in

194 ILC2s, thus explaining the unimpaired development of IL-13-dependent fibrosis in triple deficient mice by week 12 .

Disrupting TSLP, IL-25, and IL-33 signaling during acute granuloma formation

After considering the transient nature of immune control affected by disrupting the three mediators during $S$. mansoni infection, we hypothesized that the effect of blocking all three cytokines would be more apparent when applied to a more acute model where the cytokines are blocked from the onset of injury. Primary and secondary lung granuloma models were employed for these studies because they provide simple and short-term systems to dissect the importance of TSLP, IL-25, and IL-33 during both the sensitization and maintenance phases of a type 2 cytokine-driven inflammatory response (31). Groups were treated with either isotype control antibodies or with $\alpha \mathrm{TSLP}, \alpha \mathrm{IL}-25$ $\alpha \mathrm{ST} 2$ (IL-33 receptor) monoclonal neutralizing antibodies for the entire length of the experiments. The pathological effects of TSLP, IL-25, and 
IL-33 have been directly linked to the enhanced production of IL-4, IL-5, and IL-13 by downstream target cells such as $\mathrm{CD}^{+} \mathrm{Th} 2$ cells, ILC2s, and other innate lymphocytes $(33,34)$, and much of the pathology that results from the persistent activation of type 2 immunity has been attributed to IL-4/IL-13-mediated signaling through the IL-4 receptor (35). Therefore, we used IL-4R $\Gamma$-deficient mice as positive controls.

Surprisingly, as observed in previous lung granuloma studies where TSLP, IL-25, and IL33 were targeted individually, the combined blockade of all three cytokines had no significant impact on the volume of the lesions in mice undergoing either primary (Fig. 4A) or secondary (Fig. 4B) granuloma formation. In marked contrast, the lesions in IL-4R $\alpha^{-/-}$mice were about 50\% smaller than those in isotype control treated mice (Figs. 4A-B). The triple blockade did lead to a $>80 \%$ reduction in the number of granuloma-associated eosinophils during primary granuloma formation (Fig. 4A, right panel and tissue sections). Macrophages and primarily lymphocytes comprised the granulomas in the absence of eosinophils. Nevertheless, the eosinophil deficit in the triple blockade mice was completely corrected when the mice were undergoing a secondary challenge (Fig. 4B, right panel and tissue sections). IL-4R $\alpha^{-/-}$mice, in contrast, displayed a near complete absence of eosinophils following both primary and secondary 223 challenges.

Although the effects of the triple blockade on egg-induced pathology were minimal, there were notable changes in cytokine expression in the lungs. Triple blockade mice displayed significant reductions in IL-4, IL-5, and IL-13 expression in the lung during primary granuloma formation (Fig. 4C) and in IL-5 and IL-13 during secondary granuloma formation (Fig. 4D). It is worth noting that while these measurements imply a significant reduction in the type 2 cytokines following triple blockade, they were expressed at significantly higher levels than that 
in IL-4R $\alpha$-deficient mice. Interestingly, changes in expression of two eosinophilic chemokines,

231 Ccl5 and Ccl11, do not explain the eosinophil phenotype we observed. Ccl5 and Ccl11 were not

232 affected by the triple blockade during the primary response (Fig. 4C) although both chemokines

233 were reduced in the triple blockade mice during a secondary response (Fig. 4D). Rather, the

234 pattern of $I l 5$ gene expression likely explains why granuloma eosinophilia is reduced by the

235 triple blockade during primary granuloma formation and is restored during secondary granuloma

236 formation. The reduced $I l 5$ expression in triple blockade mice during primary granuloma

237 formation was on par with expression observed in IL-4R $\alpha$-deficient mice. During secondary

238 granuloma formation, $I l 5$ expression was reduced by the triple blockade, but it was still

239 expressed at significantly higher levels than in IL-4R $\alpha$-deficient mice.

As seen in many type 2 cytokine-driven diseases, we observed increased Il33, Tslp, and

241 Il25 gene expression in the lungs of wild-type mice in the primary granuloma model (Fig. 4C).

242 While gene expression of $I l 33$ and $T s l p$ increased in the lungs of mice undergoing secondary

243 granuloma formation, $I l 25$ was expressed at baseline levels during the secondary response (Fig.

244 4D). The increase was IL-4R $\alpha$-dependent as $I l 33$ and $T s l p$ expression diminished to baseline

245 levels in IL-4R $\alpha^{-/-}$mice. We hypothesize the low alarmin expression in IL-4R $\alpha^{-/-}$mice is due to

246 decreased inflammation-driven injury in these mice.

247 Together, our studies with S. mansoni demonstrated that TSLP, IL-25, and IL-33 play

248 redundant roles in the maintenance of chronic type 2 immunity. More importantly, targeting all

249 three cytokines simultaneously from the initiation of primary or secondary granuloma formation

250 reduced type 2 cytokine production but offered little protection from egg-induced pathology. 
253 With evidence that the combined blockade of TSLP, IL-25, and IL-33 signaling had a significant 254 impact on type 2 cytokine expression, we hypothesized that the triple blockade might ameliorate 255 type 2-mediated pathology in a different disease model. We sought to investigate a model that 256 primarily targets epithelial cells, the predominant source of TSLP, IL-25, and IL-33, to

257 determine whether the maintenance of type 2 immunity induced via mucosal epithelial injury 258 was more dependent on the targeted cytokines. We chose to test the effects of administering 259 single, double, and triple mAb blockades to a model of house dust mite (HDM)-induced allergic 260 inflammation entering its chronic stage. Genes for all three alarmins are expressed at steady 261 state in the lung, and HDM induces expression of each of the alarmins with complementary 262 kinetics (Fig. S2). Il33 was upregulated acutely and at chronic stages of allergic disease. Tslp 263 was only upregulated in the initial hours after first HDM exposure, and Il25 was upregulated 264 only at chronic time-points. BALB/c mice were chronically challenged i.n. with HDM on days 2650,7 , and 14 and then received eight additional doses spread over a total of 45 days. Beginning 266 three weeks after the initiation of the allergic response, separate groups of HDM-treated mice 267 were administered doses of anti-ST2, anti-TSLP, anti-IL-25 every 3 to 4 days in various 268 combinations to achieve single, double, or triple blockades. Additional control groups received 269 either saline or isotype control antibodies with or without HDM. On day 46, all mice were 270 analyzed. As expected, in the lungs of isotype-treated control mice, chronic HDM exposure 271 resulted in a marked increase in inflammatory cells in the lung (Fig. 5A) and nearly a two-fold 272 increase in collagen content (Fig. 5B), confirming extensive lung remodeling and fibrosis.

273 Surprisingly however, none of the single, double, or triple blockade combinations led to a 274 significant decrease in inflammation or fibrosis in the sensitized mice. When the triple blockade 275 mice were analyzed more closely, we also observed little to no change in the type 2 cytokine 
response in the lung (Fig. 5C), and the total number of leukocytes in the BAL and lung appeared indistinguishable between the triple blockade and isotype control treated mice (Fig. 5D). We did, however, observe a significant decrease in the percentage of eosinophils in the lung but not in the BAL (Fig. 5E).

Disrupting TSLP, IL-33, and IL-25 signaling during initiation and maintenance of type 2-

\section{driven chronic allergy}

The failure of the triple blockade to protect against type 2-driven pathology when applied to established allergy further suggested that TSLP, IL-25, and IL-33 are not critical for the maintenance of chronic type 2 driven allergic lung inflammation. To test whether disrupting signaling of the three cytokines during the initiation of type 2 cytokine-driven allergic lung inflammation provides a benefit, in a final series of experiments, IL-33/TSLP DKO mice were treated with anti-IL-25 during the entire course of chronic HDM exposure. Here, the deficient mice displayed marked and significant decreases in fibrosis when compared with control HDM mice on day 46 (Fig. 6A). Although peribronchial and perivascular inflammation in the lung was similar in both groups, we observed a marked decrease in endarteritis and mucus staining in the lumen of the deficient mice (Fig. 6B). In addition, the total number of BAL cells (Fig. 6C), and the number of eosinophils in the BAL (Fig. 6D) and lung (Fig. 6E) were reduced. The

294 decrease in inflammatory eosinophils was also accompanied by a marked and highly significant 295 reduction in IL-4, IL-5, and IL-13 production in the lung (Fig. 6F) and IL-13 and IL-5 were also 296 significantly decreased in the BAL (Fig. 6G). We observed similar results using anti-ST2, anti297 TSLP, anti-IL-25 neutralizing antibodies in wild-type mice during the entire course of chronic HDM exposure (Fig. S3). 


\section{Discussion}

Although TSLP, IL-25, and IL-33 have each been identified as important initiators of type 2 immunity, their role in the maintenance of progressive type 2 disease was much less clear. In the present study, using chronic models of helminth infection and type 2 cytokine-driven lung inflammation, we found that tri-functional targeting of TSLP, IL-25, and IL-33 was more efficacious than blocking any one of the mediators alone. This conclusion is strengthened since we made the observations using mice on both $\mathrm{C} 57 \mathrm{BL} / 6$ and $\mathrm{BALB} / \mathrm{c}$ backgrounds. In a

308 schistosome lung granuloma model or during chronic $S$. mansoni infection in the liver, selective 309 ablation of TSLP, IL-25, or IL-33/ST2 had little to no impact on the development of IL-4/IL-13dependent inflammation or fibrosis. Nevertheless, we observed modest albeit significant reductions in egg-induced inflammation in the liver when signaling of all three mediators was disrupted simultaneously. The reduction in inflammation in the schistosome infection model was also accompanied by a small yet significant decrease in the number of granuloma-associated eosinophils, a $25-30 \%$ decrease in hepatic fibrosis, and a significant reduction in the number of IL-13-producing ILC2s in the mesenteric lymph nodes. The deficient mice also displayed reduced expression of IL-5 and IL-13 during primary and secondary granuloma formation in the

317 lung. Furthermore, when signaling of all three mediators was disrupted in a model of chronic 318 HDM-induced allergic lung inflammation, inflammation, mucus production, and lung 319 remodeling were decreased. Together, these studies revealed redundant roles for TSLP, IL-25, 320 and IL-33 in the maintenance of these type 2-associated pathologies and suggest that aggressive 
tri-functional targeting of these mediators may more effectively ameliorate progressive type 2driven disease.

Previous studies identified critical roles for TSLP, IL-25, and IL-33 in type 2 immunity to some helminth parasites (36-43). However, the majority of these studies have focused on Nippostrongylus brasiliensis infection, in which expulsion of the nematode parasite is delayed or accelerated by relatively minor changes in type 2 immunity. Our initial studies focused on the schistosome lung granuloma and $S$. mansoni infection models because these models provide robust systems to dissect the role of TSLP, IL-25, and IL-33 during both the initiation and maintenance phases of type 2-driven inflammation (31). As reported previously with TSLP (26), we observed little to no role for IL-25 or IL-33 in IL-4/IL-13-dependent granuloma formation in the lung. A recent study found modest decreases in acute inflammation in the absence of IL-25 (44), but in our studies, IL-25 or IL-33 deficiency alone had no discernable impact on the development of type 2 immunity or type 2-dependent pathology, even during the initiation of a primary granulomatous response. A similar outcome was observed in the liver following acute and chronic infection with S. mansoni, suggesting that TSLP, IL-25, and IL-33 were either not required or were possibly playing redundant roles $(26,28,38,45)$. Importantly, although we found little to no role for TSLP, IL-25, or IL-33/ST2 when each mediator was ablated individually, we observed significant reductions in type 2 inflammation and fibrosis in the liver when all three mediators were targeted simultaneously, confirming their overlapping activities in response to significant damage during acute schistosomiasis. It is possible the degree of damage from parasites and other environmental triggers may impact the redundancy of the alarmins. Also, schistosome egg antigens have been identified that are capable of directly activating type 2 responses by modulating dendritic cell function $(46,47)$. Basophil- and autocrine T cell-derived 
IL-4 may also be sufficient to initiate and maintain type 2 responses $(48,49)$. Therefore, alarmins may not be critical to the activation or maintenance of all type- 2 cytokine driven inflammatory responses.

The type 2 response is a critical driver of wound repair pathways (1). However when type 2 cytokine production persists or becomes dysregulated, it can lead to the development of pathological fibrosis (3). Consequently, because of their type 2-inducing activity, there has been a great deal of interest in understanding the roles of TSLP, IL-25, and IL-33 in progressive fibrosis, with numerous studies identifying increased production of these cytokines in various fibrotic diseases (50-54). Many recent studies have shown that when overexpressed in mice, TSLP, IL-25, and IL-33 induce fibrosis in multiple tissues. For example, IL-25 was shown to promote lung remodeling in a model of house dust mite induced allergic airway disease and 355 indirectly induced pulmonary fibrosis by stimulating the production of IL-13 from ILC2s (44, 54). Transgenic overexpression of IL-33 has also been shown to promote IL-13-dependent cutaneous fibrosis (55), ILC2-mediated hepatic fibrosis (32), and bleomycin-induced pulmonary fibrosis in mice (56). Transgene-induced expression of TSLP has also been shown to induce pulmonary fibrosis in the lung by upregulating type 2 cytokine expression (52). Nevertheless, evidence that these epithelial-derived alarmins are critical to the development of $\mathrm{Th} 2$-associated

361 fibrosis in a natural model of fibrosis was lacking prior to this study. Our studies with the schistosome lung granuloma and infection models show quite unequivocally that IL-13dependent fibrosis can develop in the lung and liver independently of TSLP, IL-25, and IL-33.

364 We did, however, observe a significant decrease in fibrosis when all three mediators were 365 targeted simultaneously, with the reduction in fibrosis associated with a significant decrease in 366 IL-13 producing ILC2s. Surprisingly, at more chronic time points following infection with $S$. 
mansoni the early reduction in fibrosis and ILC2 activity appeared to be compensated for by an increased $\mathrm{CD}^{+} \mathrm{T}$ cell-derived IL-13 response, suggesting that TSLP, IL-25, IL-33 and ILC2s may not be critical to the maintenance of established and progressive fibrosis once the adaptive immune response has taken over. The relative involvement of an adaptive antigen-specific response may therefore be important in determining the relative contribution of these innate pathways to chronic disease. Regardless, these data further emphasize the potential benefit of early combinatorial targeting of TSLP, IL-25, and IL-33 in the treatment of type 2-driven disease.

Because epithelial cells are a major source of TSLP, IL-25, and IL-33 and schistosome eggs primarily damage the endothelium, it is possible that these cytokines are less important to the development of type 2 pathology in schistosomiasis. Therefore, in a final series of experiments, we utilized a chronic model of HDM-induced allergic lung inflammation to explore the combined roles of TSLP, IL-25, and IL-33 in a disease where the epithelium is the primary target. Here, in contrast to the lung granuloma studies in which a mAb triple blockade administered from initial egg challenge had little impact on type 2 pathology, disrupting TSLP, IL-25, and IL-33 signaling from first allergen exposure had a significant suppressive effect on the development of fibrosis, endarteritis, and mucus deposition in the lumen. The number of inflammatory cells in the BAL was also reduced, as were the number of eosinophils in the BAL and lung, with the reduction in eosinophils consistent with a recent study exploring the roles of TSLP, IL-25, and IL-33 in a model of chitin-induced lung inflammation (57). We also observed 387 marked and highly significant reductions in IL-5 and IL-13 production in the lung and BAL 388 fluid. When the combined mAb blockade of TSLP, IL-25, and IL-33 was applied to a model of established allergic lung inflammation, the marked protective effects were almost completely 
lost, however, suggesting that TSLP, IL-33, and IL-25 are either not required for the maintenance of an established antigen-specific type 2 response or that earlier intervention with TSLP, IL-33, and IL-25 antagonists is needed.

Although TSLP, IL-33, and IL-25 were all initially identified as critical drivers of type 2 immunity $(10,12,50)$, several subsequent studies have illustrated that type 2 immunity can develop independently of these cytokines $(24-26,28)$. The results from our experiments suggest that much of the data in the latter studies are likely explained by the overlapping activities of TSLP, IL-33, and IL-25. Our data also suggest the three alarmins may be dispensable for the maintenance of type 2 immunity and chronic type 2-associated pathology because continued exposure to complex antigens like schistosome eggs or house dust mite allergen generates a potent and sustained adaptive $\mathrm{CD}^{+}$type 2 response that can supplant the requirement for alarmins and innate lymphocytes. A recent double-blind, placebo-controlled study of AMG 157, a neutralizing anti-human TSLP mAb, showed that TSLP blockade could reduce allergeninduced bronchoconstriction and eosinophilia (58). Whether targeting TSLP alone would show clinical benefit in moderate-severe asthma, however, could not be discerned from this small study tested on allergic individuals with near-normal baseline lung function.

Differences in perturbations of epithelium and other stromal cells may dictate the relative contribution of the three alarmins, and further studies with different animal models of allergy (e.g. allergen dosing, variety, airway hypersensitivity) will be important before large-scale human studies are considered. The cost and time required for chronic models prevented us from testing all combinations of single, double, and triple blockades in every model. Notably, the triple blockade with mAbs from the start of allergic disease is effective, but its impact was not identical to congenital knockouts by all measures. Although all three antibodies were confirmed 
to exhibit highly effective neutralizing activity, it is possible that incomplete target coverage with

414 the antibodies might in part explain these differences as well as the minimal efficacy of treating

415 mice with established allergic disease. It is also possible that intracrine alarmin signaling such as

416 IL-33-mediated activation of NF- $\mathrm{KB}$ contributes to these small differences. In any case, antibody

417 target coverage should be carefully evaluated in any future study in humans. Chronic human

418 disease is likely maintained by a complex assortment of signals combined with sporadic

419 exposure to specific antigen, and a better understanding of the hierarchy of these cues will help

420 to clarify the relative contributions of TSLP, IL-33, and IL-25, as well as ILC2s. Our data

421 suggest that a strategy that simultaneously suppresses more than one of these alarmins from the

422 early phase of the disease may be required to effectively target type 2 cytokine-driven disease.

\section{Materials and Methods}

\section{Study Design}

427 Our primary objective was to investigate the effects of ablating IL-33, TSLP, and IL-25 signaling

428 on chronic type 2 inflammation and fibrosis. To do this, we developed strategies to disrupt the

429 signaling of the cytokines in mouse models of progressive type 2 immune-related pathology. No

430 statistical methods were used to predetermine sample size. Group sample size was chosen using

431 records of variance in past experiments, and variance is similar between groups being

432 statistically compared. Samples or data points were excluded only in the case of a technical

433 equipment or human error that caused a sample to be poorly controlled for. Mice or samples

434 were randomly assigned to experimental groups or processing orders. Group allocation was

435 blinded for all mouse work, when possible (e.g. administration of proteins, schistosomes, or 
436 allergens, sample quantification and analysis, pathology scoring). The ARRIVE guidelines in 437 the EQUATOR Network library were followed for this report.

\section{Animals}

440 The National Institute of Allergy and Infectious Diseases Division of Intramural Research 441 Animal Care and Use Program, as part of the National Institutes of Health Intramural Research 442 Program, approved all of the experimental procedures (protocol "LPD 16E"). The Program 443 complies with all applicable provisions of the Animal Welfare Act

444 (http://www.aphis.usda.gov/animal_welfare/downloads/awa/awa.pdf) and other federal statutes 445 and regulations relating to animals. IL-33 $3^{-/}$and IL-33/TSLP double knockout mice on a 446 C57BL/6 background were provided by Amgen Inc. C57BL/6, BALB/c, and IL-4R $\alpha^{-/-}$mice 447 were obtained from Taconic Farms Inc. IL-25 $5^{-/}$mice were obtained from Regeneron 448 Pharmaceuticals, Inc. Male and female mice between the ages of 6 weeks and 12 weeks were used randomly to begin experimental models because of limited availability, and no sex-specific

450 differences were observed. Groups in individual experiments were sex-matched and age451 matched. All animals were housed under specific pathogen-free conditions at the National 452 Institutes of Health in an American Association for the Accreditation of Laboratory Animal 453 Care-approved facility.

\section{Parasite infection}

456 Mice were infected percutaneously via the tail with 35 cercaria from a Puerto Rican strain of 457 Schistosoma mansoni (NMRI) obtained from infected Biomphalaria glabrata snails (Biomedical 458 Research Institute). 35 cercaria infection in wild-type mice leads to substantial disease and liver 
460 of euthanasia to determine worm and tissue egg burdens as described previously (59).

\section{Chronic house dust mite-induced allergy}

463 Mice anesthetized with isoflurane were challenged intranasally with $200 \mu \mathrm{g}$ of house dust mite

464 (HDM) in $30 \mu 1$ saline on days 0,7 , and 14 followed by eight additional $50 \mu \mathrm{g}$ doses in $30 \mu 1$

465 saline spread over a total of 45 days. Lungs were harvested on day 46.

\section{Schistosome egg-induced lung granuloma models}

468 For the primary lung granuloma model, 5000 live $S$. mansoni eggs (Biomedical Research

469 Institute) in saline were injected intravenously into mice on day 0. Lungs were harvested on day

4707 for analysis. For the secondary lung granuloma model, 5000 S. mansoni eggs were also injected intraperitoneally on day 0 . Mice were injected intravenously with 5000 live eggs containing mature embryos again on day 14 before lungs were harvested on day 21 .

\section{Hydrodynamic delivery of IL-25}

Mice were injected intravenously with $10 \mu \mathrm{g}$ of a mammalian expression plasmid coding for murine IL-25 in $2 \mathrm{ml}$ of warm saline (60).

Triple block of IL-33, TSLP, and IL-25 with monoclonal antibodies

479 Anti-mouse ST2 (61), anti-mouse TSLP (38), and anti-mouse IL-25 (62) monoclonal antibodies

480 were generated and selected by Amgen Inc. after extensive in vitro and in vivo testing.

481 Previously unpublished tests for the efficacy of anti-TSLP included a bone marrow-derived 
dendritic cell bioassay measuring the inhibition of TSLP-induced CCL17/TARC production and an assay measuring inhibition of TSLP-induced proliferation of a pro-B cell line stably transduced with murine TSLP receptor. Neutralization of IL-33, TSLP, and IL-25 signaling was achieved by administering $250 \mu \mathrm{g}$ of these antibodies, respectively, via intraperitoneal injection twice-weekly. To properly control for the neutralizing antibodies, groups administered single and double blocks also received $250 \mu \mathrm{g}$ mouse IgG1 in the absence of anti-ST2 or anti-IL-25, and $250 \mathrm{Og}$ rat IgG1 in the absence of anti-TSLP.

\section{Histopathology}

Liver or lung tissue was fixed in Bouin-Hollande solution, embedded in paraffin for sectioning, and stained (Histopath of America) with Wright's Giemsa (S. mansoni models), hematoxylin and eosin, or Masson's trichrome (allergy model) for analysis of inflammation, picrosirius red or Masson's trichrome for fibrosis analysis, or Periodic acid-Schiff (PAS) stain for analysis of mucus production. A scale of 1 to 4 ( 4 being the highest) was used for scoring. A blinded pathologist measured the diameter of approximately 30 granulomas and quantified granulomatous eosinophils in Giemsa-stained sections of each sample with granulomatous pathology. Images were scanned with an Aperio ScanScope (Leica Biosystems).

\section{Fibrosis assay}

Hydroxyproline was measured as a surrogate for collagen content. A known weight of liver or lung tissue was hydrolyzed in $6 \mathrm{~N} \mathrm{HCl}$ at $110^{\circ} \mathrm{C}$ for $18 \mathrm{~h}$ and then neutralized in $10 \mathrm{~N} \mathrm{NaOH}$ before colorization. A standard curve comprised of dilutions of 1mM hydroxyproline (SigmaAldrich) (63). 


\section{Leukocyte isolation for intracellular cytokine staining and eosinophil identification}

About $200 \mathrm{mg}$ of lung or liver tissue was ground into a single-cell suspension through a $100-\mu \mathrm{m}$ nylon mesh. Leukocytes were separated on a 40\% Percoll (Sigma-Aldrich) gradient (2000 rpm for $15 \mathrm{~min}$ ) and treated for $2 \mathrm{~min}$ with $1 \mathrm{ml} \mathrm{ACK}$ (ammonium chloride-potassium bicarbonate) lysis buffer to lyse erythrocytes. After 3 hours of stimulation with phorbol 12-myristate 13acetate (PMA 10ng/ml), ionomycin $(1 \mu \mathrm{g} / \mathrm{ml})$, and Brefeldin A (BFA, $10 \mu \mathrm{g} / \mathrm{ml})$, leukocytes were fixed and permeabilized for 30 minutes (Cytofix/Cytoperm buffer; BD Biosciences) and then

513 stained for 30 minutes with antibodies for CD4 (Clone: RM4-5; eBioscience), IFN- $\gamma$ (XMG1.2,

514 eBioscience), IL-4 (11B11, eBioscience), IL-5 (TRFK5, BD Pharmingen), and IL-13 (eBio13A,

515 eBioscience) diluted in the Permwash buffer (BD Biosciences). Unstimulated lung leukocyte

516 aliquots were set aside and stained for 30 minutes with anti-SiglecF. Postive SiglecF staining and 517 scatter profiling were used to identify eosinophils by flow cytometry. Leukocytes collected from 518 bronchoalveolar lavage were isolated with ACK lysis buffer, stimulated, fixed, permeabilized, 519 and stained as above. Expression of CD4, SiglecF, and the intracellular cytokines was analyzed 520 with a BD FACSCanto II flow cytometer and FlowJo v.7.6 software (Tree Star).

\section{Leukocyte isolation from liver and mesenteric lymph node for ILC2 staining}

523 Liver or lymph node tissue was ground into a single-cell suspension through a $100-\mu \mathrm{m}$ nylon 524 mesh, and hepatic leukocytes required further separation using a 40\% Percoll gradient and ACK 525 lysis as described above. Leukocyte samples from both tissues were stimulated, fixed, and 526 permeabilized as described above. Then they were stained for 30 minutes with antibodies for 527 CD16/32 (Clone: 2.4G2, BDBiosciences), CD4 (RM4-5, eBioscience), IL-13 (eBio13A, 
eBioscience), ST2 (DJ8, MD Biosciences), and ICOS (C398.4A, Biolegend) diluted in Permwash buffer (BD Biosciences). Expression of the surface markers and intracellular IL-13 was analyzed with a BD FACSCanto II flow cytometer and FlowJo v.7.6 software (Tree Star).

\section{RNA isolation and quantitative real-time PCR}

Lung or liver tissue was homogenized in TRIzol Reagent (Life Technologies) using Precellys 24 (Bertin Technologies). Total RNA was extracted from the homogenate by addition of chloroform followed by the recommendations of the MagMax-96 Total RNA Isolation Kit (Life Technologies). RNA was then reverse transcribed using SuperScript II Reverse Transcriptase

537 (Life Technologies). Real-time RT-PCR was performed on an ABI Prism 7900HT Sequence 538 Detection System (Applied Biosystems). Quantities of mRNA expressed by a particular gene 539 were determined using Power SYBR Green PCR Master Mix (Applied Biosystems), normalized 540 to ribosomal protein, large, P2 (RPLP2) mRNA levels in each sample, and then articulated as a 541 relative increase or decrease compared with mRNA levels expressed by the same gene in naive controls. Primers were designed using Primer Express software (version 2.0; Applied

543 Biosystems). Forward and reverse primer sequences are listed in Table S1.

\section{Bronchoalveolar lavage, cell differential determination, and ELISA}

$5461 \mathrm{ml}$ of ice-cold PBS supplemented with 5mM EDTA was injected through the trachea into the

547 lungs and aspirated using a syringe. $\sim 1 \times 10^{5}$ cells were spun for 5 mins with a Shandon Cytospin 5483 centrifuge (Thermo Scientific) onto a slide before being fixed with methanol and stained with 549 Diff-Quik (Boehringer) to identify leukocyte cell-types. Levels of IL-4, IL-5, and IL-13 in the 
undiluted BAL were quantified using a Luminex-based multiplex assay according to manufacturer's protocol (EMDMillipore).

\section{Statistical analysis}

554

555

567 1. W. C. Gause, T. A. Wynn, J. E. Allen, Type 2 immunity and wound healing: evolutionary

All data were analyzed with Prism (Version 5; GraphPad). Data sets were compared with a twotailed t-test, and differences were considered significant if $P$ values were less than 0.05 . A Welch's correction was used when an F-test comparing variances had a $P$ value of less than 0.05 .

\section{Supplementary Materials}

Fig. S1. Alarmin gene expression in the liver.

Fig. S2. Kinetics of alarmin gene expression in chronic HDM model.

Fig. S3. Neutralizing all three alarmins with mAbs during initiation and maintenance of type 2driven allergy reduces inflammation and fibrosis.

Table S1. qPCR Primer Sequences.

\section{References} refinement of adaptive immunity by helminths. Nature reviews. Immunology 13, 607-614 (2013).

2. A. K. Abbas, K. M. Murphy, A. Sher, Functional diversity of helper T lymphocytes. Nature 383, 787-793 (1996). 
3. T. A. Wynn, Fibrotic disease and the $\mathrm{T}(\mathrm{H}) 1 / \mathrm{T}(\mathrm{H}) 2$ paradigm. Nature reviews. Immunology 4, 583-594 (2004).

4. A. Chawla, K. D. Nguyen, Y. P. Goh, Macrophage-mediated inflammation in metabolic disease. Nature reviews. Immunology 11, 738-749 (2011).

5. J. E. Allen, R. M. Maizels, Diversity and dialogue in immunity to helminths. Nature reviews. Immunology 11, 375-388 (2011).

6. H. Y. Kim, R. H. DeKruyff, D. T. Umetsu, The many paths to asthma: phenotype shaped by innate and adaptive immunity. Nature immunology 11, 577-584 (2010).

7. G. Bouma, W. Strober, The immunological and genetic basis of inflammatory bowel disease. Nature reviews. Immunology 3, 521-533 (2003).

582 8. F. Y. Liew, N. I. Pitman, I. B. McInnes, Disease-associated functions of IL-33: the new kid in the IL-1 family. Nature reviews. Immunology 10, 103-110 (2010).

5849 9. T. A. Wynn, T. R. Ramalingam, Mechanisms of fibrosis: therapeutic translation for 585 fibrotic disease. Nature medicine 18, 1028-1040 (2012).

10. M. M. Fort, J. Cheung, D. Yen, J. Li, S. M. Zurawski, S. Lo, S. Menon, T. Clifford, B. Hunte, R. Lesley, T. Muchamuel, S. D. Hurst, G. Zurawski, M. W. Leach, D. M. Gorman, D. M. Rennick, IL-25 induces IL-4, IL-5, and IL-13 and Th2-associated pathologies in vivo. Immunity 15, 985-995 (2001).

590 11. V. Soumelis, P. A. Reche, H. Kanzler, W. Yuan, G. Edward, B. Homey, M. Gilliet, S. Ho, S. Antonenko, A. Lauerma, K. Smith, D. Gorman, S. Zurawski, J. Abrams, S. Menon, T. McClanahan, R. de Waal-Malefyt Rd, F. Bazan, R. A. Kastelein, Y. J. Liu, Human epithelial cells trigger dendritic cell mediated allergic inflammation by producing TSLP. Nature immunology 3, 673-680 (2002). 
12. J. Schmitz, A. Owyang, E. Oldham, Y. Song, E. Murphy, T. K. McClanahan, G. Zurawski, M. Moshrefi, J. Qin, X. Li, D. M. Gorman, J. F. Bazan, R. A. Kastelein, IL-33, an interleukin-1-like cytokine that signals via the IL-1 receptor-related protein ST2 and induces T helper type 2-associated cytokines. Immunity 23, 479-490 (2005).

13. C. A. Christianson, N. P. Goplen, I. Zafar, C. Irvin, J. T. Good, Jr., D. R. Rollins, B. Gorentla, W. Liu, M. M. Gorska, H. Chu, R. J. Martin, R. Alam, Persistence of asthma requires multiple feedback circuits involving type 2 innate lymphoid cells and IL-33. $J$ Allergy Clin Immunol 136, 59-68 e14 (2015).

14. A. Shikotra, D. F. Choy, C. M. Ohri, E. Doran, C. Butler, B. Hargadon, M. Shelley, A. R. Abbas, C. D. Austin, J. Jackman, L. C. Wu, L. G. Heaney, J. R. Arron, P. Bradding, Increased expression of immunoreactive thymic stromal lymphopoietin in patients with severe asthma. J Allergy Clin Immunol 129, 104-111 e101-109 (2012).

15. D. Cheng, Z. Xue, L. Yi, H. Shi, K. Zhang, X. Huo, L. R. Bonser, J. Zhao, Y. Xu, D. J. Erle, G. Zhen, Epithelial interleukin-25 is a key mediator in Th2-high, corticosteroidresponsive asthma. American journal of respiratory and critical care medicine 190, 639648 (2014).

16. P. Licona-Limon, L. K. Kim, N. W. Palm, R. A. Flavell, TH2, allergy and group 2 innate lymphoid cells. Nature immunology 14, 536-542 (2013).

17. M. D. Smithgall, M. R. Comeau, B. R. Yoon, D. Kaufman, R. Armitage, D. E. Smith, IL33 amplifies both Th1- and Th2-type responses through its activity on human basophils, allergen-reactive Th2 cells, iNKT and NK cells. Int Immunol 20, 1019-1030 (2008).

18. M. R. Comeau, S. F. Ziegler, The influence of TSLP on the allergic response. Mucosal immunology 3, 138-147 (2010). 
19. J. Mjosberg, J. Bernink, K. Golebski, J. J. Karrich, C. P. Peters, B. Blom, A. A. te Velde, W. J. Fokkens, C. M. van Drunen, H. Spits, The transcription factor GATA3 is essential for the function of human type 2 innate lymphoid cells. Immunity 37, 649-659 (2012).

20. T. Y. Halim, R. H. Krauss, A. C. Sun, F. Takei, Lung natural helper cells are a critical source of Th2 cell-type cytokines in protease allergen-induced airway inflammation. Immunity 36, 451-463 (2012).

21. B. S. Kim, M. C. Siracusa, S. A. Saenz, M. Noti, L. A. Monticelli, G. F. Sonnenberg, M. R. Hepworth, A. S. Van Voorhees, M. R. Comeau, D. Artis, TSLP elicits IL-33independent innate lymphoid cell responses to promote skin inflammation. Science translational medicine 5, 170ra116 (2013).

22. N. Lu, Y. H. Wang, Y. H. Wang, K. Arima, S. Hanabuchi, Y. J. Liu, TSLP and IL-7 use two different mechanisms to regulate human CD4+ T cell homeostasis. $J$ Exp Med 206, 2111-2119 (2009).

23. N. Murakami-Satsutani, T. Ito, T. Nakanishi, N. Inagaki, A. Tanaka, P. T. Vien, K. Kibata, M. Inaba, S. Nomura, IL-33 promotes the induction and maintenance of Th2 immune responses by enhancing the function of OX40 ligand. Allergol Int 63, 443-455 (2014).

24. M. A. Willart, K. Deswarte, P. Pouliot, H. Braun, R. Beyaert, B. N. Lambrecht, H. Hammad, Interleukin-1alpha controls allergic sensitization to inhaled house dust mite via the epithelial release of GM-CSF and IL-33. J Exp Med 209, 1505-1517 (2012).

25. S. Jang, S. Morris, N. W. Lukacs, TSLP promotes induction of Th2 differentiation but is not necessary during established allergen-induced pulmonary disease. PloS one 8, e56433 (2013). 
26. T. R. Ramalingam, J. T. Pesce, M. M. Mentink-Kane, S. Madala, A. W. Cheever, M. R. Comeau, S. F. Ziegler, T. A. Wynn, Regulation of helminth-induced Th2 responses by thymic stromal lymphopoietin. J Immunol 182, 6452-6459 (2009).

27. K. Oboki, T. Ohno, N. Kajiwara, K. Arae, H. Morita, A. Ishii, A. Nambu, T. Abe, H. amplifier of innate rather than acquired immunity. Proc Natl Acad Sci U S A 107, 18581$18586(2010)$.

28. J. C. Massacand, R. C. Stettler, R. Meier, N. E. Humphreys, R. K. Grencis, B. J. Marsland, N. L. Harris, Helminth products bypass the need for TSLP in Th2 immune responses by directly modulating dendritic cell function. Proc Natl Acad Sci US A 106, 13968-13973 (2009).

652

29. J. M. Mjosberg, S. Trifari, N. K. Crellin, C. P. Peters, C. M. van Drunen, B. Piet, W. J. Fokkens, T. Cupedo, H. Spits, Human IL-25- and IL-33-responsive type 2 innate lymphoid cells are defined by expression of CRTH2 and CD161. Nature immunology 12, 1055-1062 (2011).

30. S. A. Saenz, B. C. Taylor, D. Artis, Welcome to the neighborhood: epithelial cell-derived cytokines license innate and adaptive immune responses at mucosal sites. Immunological reviews 226, 172-190 (2008).

659 31. T. A. Wynn, I. Eltoum, I. P. Oswald, A. W. Cheever, A. Sher, Endogenous interleukin 12 (IL-12) regulates granuloma formation induced by eggs of Schistosoma mansoni and exogenous IL-12 both inhibits and prophylactically immunizes against egg pathology. $J$ Exp Med 179, 1551-1561 (1994). 
32. T. McHedlidze, M. Waldner, S. Zopf, J. Walker, A. L. Rankin, M. Schuchmann, D. Voehringer, A. N. McKenzie, M. F. Neurath, S. Pflanz, S. Wirtz, Interleukin-33dependent innate lymphoid cells mediate hepatic fibrosis. Immunity 39, 357-371 (2013).

33. J. A. Walker, J. L. Barlow, A. N. McKenzie, Innate lymphoid cells--how did we miss them? Nature reviews. Immunology 13, 75-87 (2013).

34. S. J. Galli, N. Borregaard, T. A. Wynn, Phenotypic and functional plasticity of cells of innate immunity: macrophages, mast cells and neutrophils. Nature immunology 12, 10351044 (2011).

35. S. J. Van Dyken, R. M. Locksley, Interleukin-4- and interleukin-13-mediated alternatively activated macrophages: roles in homeostasis and disease. Annual review of immunology 31, 317-343 (2013).

36. A. Zhao, J. F. Urban, Jr., R. Sun, J. Stiltz, M. Morimoto, L. Notari, K. B. Madden, Z. Yang, V. Grinchuk, T. R. Ramalingam, T. A. Wynn, T. Shea-Donohue, Critical role of IL-25 in nematode infection-induced alterations in intestinal function. $J$ Immunol 185, 6921-6929 (2010).

37. C. Zaph, A. E. Troy, B. C. Taylor, L. D. Berman-Booty, K. J. Guild, Y. Du, E. A. Yost, A. D. Gruber, M. J. May, F. R. Greten, L. Eckmann, M. Karin, D. Artis, Epithelial-cellintrinsic IKK-beta expression regulates intestinal immune homeostasis. Nature 446, $552-$ $556(2007)$.

38. B. C. Taylor, C. Zaph, A. E. Troy, Y. Du, K. J. Guild, M. R. Comeau, D. Artis, TSLP regulates intestinal immunity and inflammation in mouse models of helminth infection and colitis. J Exp Med 206, 655-667 (2009). 
39. A. M. Owyang, C. Zaph, E. H. Wilson, K. J. Guild, T. McClanahan, H. R. Miller, D. J. Cua, M. Goldschmidt, C. A. Hunter, R. A. Kastelein, D. Artis, Interleukin 25 regulates type 2 cytokine-dependent immunity and limits chronic inflammation in the gastrointestinal tract. J Exp Med 203, 843-849 (2006).

40. D. R. Neill, S. H. Wong, A. Bellosi, R. J. Flynn, M. Daly, T. K. Langford, C. Bucks, C. M. Kane, P. G. Fallon, R. Pannell, H. E. Jolin, A. N. McKenzie, Nuocytes represent a new innate effector leukocyte that mediates type-2 immunity. Nature 464, 1367-1370 (2010).

41. L. Y. Hung, I. P. Lewkowich, L. A. Dawson, J. Downey, Y. Yang, D. E. Smith, D. R. Herbert, IL-33 drives biphasic IL-13 production for noncanonical Type 2 immunity against hookworms. Proc Natl Acad Sci U S A 110, 282-287 (2013).

42. N. E. Humphreys, D. Xu, M. R. Hepworth, F. Y. Liew, R. K. Grencis, IL-33, a potent inducer of adaptive immunity to intestinal nematodes. J Immunol 180, 2443-2449 (2008).

43. P. G. Fallon, S. J. Ballantyne, N. E. Mangan, J. L. Barlow, A. Dasvarma, D. R. Hewett, A. McIlgorm, H. E. Jolin, A. N. McKenzie, Identification of an interleukin (IL)-25dependent cell population that provides IL-4, IL-5, and IL-13 at the onset of helminth expulsion. J Exp Med 203, 1105-1116 (2006).

44. E. Hams, M. E. Armstrong, J. L. Barlow, S. P. Saunders, C. Schwartz, G. Cooke, R. J. Fahy, T. B. Crotty, N. Hirani, R. J. Flynn, D. Voehringer, A. N. McKenzie, S. C. Donnelly, P. G. Fallon, IL-25 and type 2 innate lymphoid cells induce pulmonary fibrosis. Proc Natl Acad Sci U S A 111, 367-372 (2014).

45. Y. H. Wang, T. Ito, B. Homey, N. Watanabe, R. Martin, C. J. Barnes, B. W. McIntyre, M. Gilliet, R. Kumar, Z. Yao, Y. J. Liu, Maintenance and polarization of human TH2 
central memory T cells by thymic stromal lymphopoietin-activated dendritic cells. Immunity 24, 827-838 (2006).

46. S. Steinfelder, J. F. Andersen, J. L. Cannons, C. G. Feng, M. Joshi, D. Dwyer, P. Caspar, P. L. Schwartzberg, A. Sher, D. Jankovic, The major component in schistosome eggs responsible for conditioning dendritic cells for Th2 polarization is a $\mathrm{T} 2$ ribonuclease

47. G. Schramm, K. Mohrs, M. Wodrich, M. J. Doenhoff, E. J. Pearce, H. Haas, M. Mohrs,

48. N. Noben-Trauth, J. Hu-Li, W. E. Paul, IL-4 secreted from individual naive CD4+ T cells acts in an autocrine manner to induce Th2 differentiation. European journal of immunology 32, 1428-1433 (2002).

49. D. Jankovic, M. C. Kullberg, N. Noben-Trauth, P. Caspar, W. E. Paul, A. Sher, Single cell analysis reveals that IL-4 receptor/Stat6 signaling is not required for the in vivo or in vitro development of CD4+ lymphocytes with a Th2 cytokine profile. J Immunol 164, 3047-3055 (2000).

50. B. Zhou, M. R. Comeau, T. De Smedt, H. D. Liggitt, M. E. Dahl, D. B. Lewis, D. Gyarmati, T. Aye, D. J. Campbell, S. F. Ziegler, Thymic stromal lymphopoietin as a key initiator of allergic airway inflammation in mice. Nat Immunol 6, 1047-1053 (2005).

51. K. Yanaba, A. Yoshizaki, Y. Asano, T. Kadono, S. Sato, Serum IL-33 levels are raised in patients with systemic sclerosis: association with extent of skin sclerosis and severity of pulmonary fibrosis. Clinical rheumatology 30, 825-830 (2011). 
52. A. Usategui, G. Criado, E. Izquierdo, M. J. Del Rey, P. E. Carreira, P. Ortiz, W. J. Leonard, J. L. Pablos, A profibrotic role for thymic stromal lymphopoietin in systemic sclerosis. Annals of the rheumatic diseases 72, 2018-2023 (2013).

53. P. Santulli, M. Even, S. Chouzenoux, A. E. Millischer, B. Borghese, D. de Ziegler, F. Batteux, C. Chapron, Profibrotic interleukin-33 is correlated with uterine leiomyoma tumour burden. Human reproduction 28, 2126-2133 (2013).

54. L. G. Gregory, C. P. Jones, S. A. Walker, D. Sawant, K. H. Gowers, G. A. Campbell, A. N. McKenzie, C. M. Lloyd, IL-25 drives remodelling in allergic airways disease induced by house dust mite. Thorax $\mathbf{6 8}, 82-90$ (2013).

55. A. L. Rankin, J. B. Mumm, E. Murphy, S. Turner, N. Yu, T. K. McClanahan, P. A. Bourne, R. H. Pierce, R. Kastelein, S. Pflanz, IL-33 induces IL-13-dependent cutaneous fibrosis. J Immunol 184, 1526-1535 (2010).

56. I. G. Luzina, P. Kopach, V. Lockatell, P. H. Kang, A. Nagarsekar, A. P. Burke, J. D. Hasday, N. W. Todd, S. P. Atamas, Interleukin-33 potentiates bleomycin-induced lung injury. American journal of respiratory cell and molecular biology 49, 999-1008 (2013).

57. S. J. Van Dyken, A. Mohapatra, J. C. Nussbaum, A. B. Molofsky, E. E. Thornton, S. F. Ziegler, A. N. McKenzie, M. F. Krummel, H. E. Liang, R. M. Locksley, Chitin activates parallel immune modules that direct distinct inflammatory responses via innate lymphoid type 2 and gammadelta T cells. Immunity 40, 414-424 (2014).

58. G. M. Gauvreau, P. M. O'Byrne, L. P. Boulet, Y. Wang, D. Cockcroft, J. Bigler, J. M. FitzGerald, M. Boedigheimer, B. E. Davis, C. Dias, K. S. Gorski, L. Smith, E. Bautista, M. R. Comeau, R. Leigh, J. R. Parnes, Effects of an anti-TSLP antibody on allergen- 

(2014).

59. A. W. Cheever, M. E. Williams, T. A. Wynn, F. D. Finkelman, R. A. Seder, T. M. Cox, S. Hieny, P. Caspar, A. Sher, Anti-IL-4 treatment of Schistosoma mansoni-infected mice inhibits development of $\mathrm{T}$ cells and non-B, non-T cells expressing Th2 cytokines while decreasing egg-induced hepatic fibrosis. J Immunol 153, 753-759 (1994).

60. T. Suda, D. Liu, Hydrodynamic gene delivery: its principles and applications. Molecular therapy : the journal of the American Society of Gene Therapy 15, 2063-2069 (2007).

61. G. Palmer, D. Talabot-Ayer, C. Lamacchia, D. Toy, C. A. Seemayer, S. Viatte, A. Finckh, D. E. Smith, C. Gabay, Inhibition of interleukin-33 signaling attenuates the severity of experimental arthritis. Arthritis Rheum 60, 738-749 (2009).

62. E. A. Rickel, L. A. Siegel, B. R. Yoon, J. B. Rottman, D. G. Kugler, D. A. Swart, P. M. Anders, J. E. Tocker, M. R. Comeau, A. L. Budelsky, Identification of functional roles for both IL-17RB and IL-17RA in mediating IL-25-induced activities. $J$ Immunol 181, 4299-4310 (2008).

63. T. A. Wynn, L. Barron, R. W. Thompson, S. K. Madala, M. S. Wilson, A. W. Cheever, T. Ramalingam, Quantitative assessment of macrophage functions in repair and fibrosis. Current protocols in immunology / edited by John E. Coligan ... [et al.] Chapter 14, Unit14 22 (2011).

Acknowledgments: We thank the NIH animal facilities for their conscientious care of mice and Amgen Inc. and Regeneron Pharmaceuticals Inc. for providing mice. 
Funding: This research was supported by the Intramural Research Program of the National Institutes of Health, National Institute of Allergy and Infectious Disease.

Author contributions: KMV TRR MRC DES TAW conceived and designed the experiments; KMV TRR AWC LAB KMH RWT SW performed the experiments; KMV TRR LAB LB KMH KNK MRC DES TAW analyzed the data; KMH analyzed the statistics; AWC ALB MRC DES contributed reagents/materials/analysis tools; KMV TAW wrote the paper.

Competing interests: ALB, MRC, DES work for a for-profit company.

Data and materials availability: Genes of interest can be accessed in NCBI's GenBank with the following codes: Rplp2: NM_026020, Il4: NM_021283, Il5: NM_010558, Il13: NM_008355,Ifn $\gamma:$ NM_008337, Il25: NM_080729, Il13: NM_008355, Ccl5: NM_013653, Ccl11:NM_011330,Il33: NM_001164724, Tslp: NM_021367.

786

Figures Legends

788

Figure 1. Ablating IL-25 offers no protection against type 2-mediated pathology.

A. Quantitative PCR analysis of gene expression in lung and liver tissue from wild type C57BL/6 mice seven days after hydrodynamic injection of IL-25 $(n=5$ mice) or PBS $(n=2)$. B. Histopathology analysis of livers from wild type and IL-13R $\Gamma 1^{-/-}$mice seven days after $S$. mansoni egg exposure and 8 days after hydrodynamic injection of IL-25 or PBS ( $n=12-15$ per group; pooled from two independent experiments; scale bars $=50 \mu \mathrm{m})$. C. Cytokine quantification from bronchoalveor lavage fluid (BALF) of mice in B ( $n=4-5$ per group). D. Histopathology analysis of lungs from IL-25 $5^{-/}$mice and littermate controls 7 days after challenge with $S$. mansoni eggs with $\left(2^{\circ}\right)$ or without priming $\left(1^{\circ}\right)$ with 5000 S. mansoni eggs 14 days prior to 

experiments for each of the models.

challenge (granuloma volume $1^{\mathrm{o}}: n=18-23$ per genotype pooled from three experiments; granuloma volume $2^{\circ}: n=9-10$ per genotype pooled from two experiments; eosinophils: $n=5$ per genotype). E. Histopathology analysis and fibrosis quantificiation of livers of IL-25 $25^{-/}$mice and littermate controls 12 weeks after infection with $S$. mansoni cercariae ( $n=9$ per genotype). A Student's t-test was used to measure all $P$ values, and $P>0.05$ except where reported. Error bars represent standard error of the mean and each data point represents a value for an individual mouse. Data are representative of two independent experiments unless otherwise noted.

\section{Figure 2. Ablating IL-33 offers no protection against type 2-mediated pathology.}

A. Fibrosis quantification and histopathology analysis of lungs from wild type C57BL/6 and IL$33^{-/-}$mice 7 days after challenge with $S$. mansoni eggs ( $n=7-10$ per genotype). B. Fibrosis quantification and histopathology analysis of lungs of the same mouse strains 21 days after priming with $S$. mansoni eggs and 7 days after challenge with eggs ( $n=10$ per genotype). C.

Fibrosis quantification of livers from the same mouse strains infected with $S$. mansoni cercariae ( $n=7-10$ per genotype). D. Micrographs of representative liver tissue sections of mice in C collected 9 weeks after infection and stained with picrosirius red (scale bar $=100 \mu \mathrm{m}) . \mathrm{E}$. Histopathology analysis of livers from the mice in $\mathrm{C}(n=7-10$ per genotype). F. Intracellular cytokine analysis of lymphocytes isolated from livers of mice in $\mathrm{C}$ nine weeks after infection measured by flow cytometry ( $n=8$ per genotype). A Student's t-test was used to measure all $P$ values, and $P>0.05$ except where reported. Data are representative of two independent 
Figure 3. Disruption of all three mediators simultaneously has a transient effect on Th2 pathology driven by S. mansoni.

A. Granuloma measurement ( $n=14-19$ per group pooled from two independent experiments) from livers of $S$. mansoni-infected wild type C57BL/6 mice administered isotype control antibody and IL-33/TSLP double knockout (DKO) mice administered anti-IL-25. B. Fibrosis quantification from livers of infected mice ( $n=7-10$ per group). C. Quantification of granuloma eosinophils from livers of infected mice ( $n=7-9$ per group). D. Quantification of CD4- IL-13$\mathrm{ST}^{+} \mathrm{ICOS}^{+}$leukocytes from the mesenteric lymph nodes (MLNs; $n=7$ per group) and livers (week 9: $n=7-8$ per group; week 12: $n=14-15$ per group pooled from two independent experiments) of infected mice by flow cytometry. E. Micrographs of representative liver tissue sections of mice 12 weeks after infection and stained with picrosirius red (scale bar $=500 \mu \mathrm{m})$. F. Intracellular cytokine analysis of liver lymphocytes of infected mice by flow cytometry $(n=14-$ 17 per genotype pooled from two experiments). A Student's t-test was used to measure all $P$ values, and $P>0.05$ except where reported. Data are representative of two independent experiments.

\section{Figure 4. Combined TSLP, IL-25, and ST2 mAb blockade during granuloma generation} diminishes type 2 immunity but not pathology.

A. Histopathology analysis of wild type BALB/c and IL-4R ${ }^{-/-}$mice seven days after injection with S. mansoni eggs. Wild type egg-injected mice were either intraperitoneally (IP) administered anti-ST2, anti-TSLP, and anti-IL-25, or corresponding isotype control antibodies ( $n=8-9$ per group). Micrographs are of representative lung sections stained with Masson's trichrome (scale bars $=50 \mu \mathrm{m})$. C. Quantification of gene expression in lung tissue from mice in 
843 A assayed by qPCR and shown relative to expression in lungs of naïve BALB/c mice $(n=3)$. B. 844 Histopathology analysis of wild type BALB/c and IL-4R ${ }^{-/}$mice seven days after injection with 845 S. mansoni eggs and 21 days after priming with $S$. mansoni eggs (Isotypes IP, Triple block IP: $n$ $846=8-9$ per group; IL-4R $\mathrm{R}^{--}: n=5$ ). Wild type egg-injected mice were either intraperitoneally 847 administered anti-ST2, anti-TSLP, and anti-IL-25, or corresponding isotype control antibodies 848 for all three weeks. Micrographs are of representative lung sections stained with Masson's 849 trichrome (scale bars $=50 \mu \mathrm{m})$. D. Quantification of gene expression in lung tissue from mice in 850 B assayed by qPCR and compared to a different group of naïve BALB/c controls $(n=3)$. A 851 Student's t-test was used to measure all $P$ values, and $P>0.05$ except where reported. Data are 852 representative of two independent experiments.

853

854 Figure 5. Efficacy of TSLP, IL-25, and ST2 mAb blockade on established chronic allergy.

855 Wild type BALB/c mice were sensitized and challenged intranasally with house dust mite 856 (HDM), and starting on day 21, anti-ST2, anti-TSLP, and/or anti-IL-25 were administered in 857 various combinations to different groups to achieve single, double, or triple blocks. Additional 858 control groups received only isotype control antibodies with or without HDM. To properly 859 control for the triple blockade group, groups administered single and double blocks also received 860 IgG1 in the absence of anti-ST2 or anti-IL-25, and rat IgG1 in the absence of anti-TSLP. All 861 mice were analyzed on day 46. A. Histopathology analysis of lung sections stained with 862 Masson's trichrome and scored for peribronchial and perivascular inflammation $(n=6-10$ per 863 group pooled from two experiments). B. Quantification of fibrosis from lung tissue. C.

864 Quantification of gene expression from lung tissue measured by qPCR. D. Quantification of 865 leukocytes in the BALF and lung tissue. E. Quantification of eosinophils shown as a percentage 
of total inflammatory cells in BALF and lung tissue. Student's t-test was used to measure all $P$ values, and $P>0.05$ except where reported.

\section{Figure 6. Disruption of all three mediators during initiation and maintenance of type 2-} driven chronic allergy reduces inflammation and fibrosis.

Wild type C57BL/6 and IL-33/TSLP DKO mice were sensitized and challenged intranasally with HDM over 45 days. DKO mice were IP administered $\alpha$ IL-25 (DKO $+\alpha$ IL-25/HDM), and HDMtreated wild-type C57BL/6 mice were IP administered an IgG1 isotype control (Isotype/HDM). A control group of C57BL/6 mice received intranasal saline instead of HDM and the isotype (Isotype/Saline). All mice were analyzed on day 46. A. Quantification of fibrosis from lung tissue (Isotype/Saline: $n=5$; Isotype/HDM: $n=9$; Triple block/Saline: $n=8$ ). B. Histopathology analysis of lung sections stained with Masson's trichrome for scoring of inflammation and ABPAS for mucus scoring. Micrographs are of representative lung sections stained with Masson's trichrome (scale bars $=50 \mu \mathrm{m}$ ). C. Quantification of leukocytes in the BALF. D. BALF leukocyte differential. E. Quantification of eosinophils in lung tissue. F. Intracellular cytokine quantification of lung tissue lymphocytes by flow cytometry. G. Intracellular cytokine quantification of BALF lymphocytes by flow cytometry. A Student's t-test was used to measure all $P$ values, and $P>0.05$ except where reported. Data are representative of two independent experiments. 


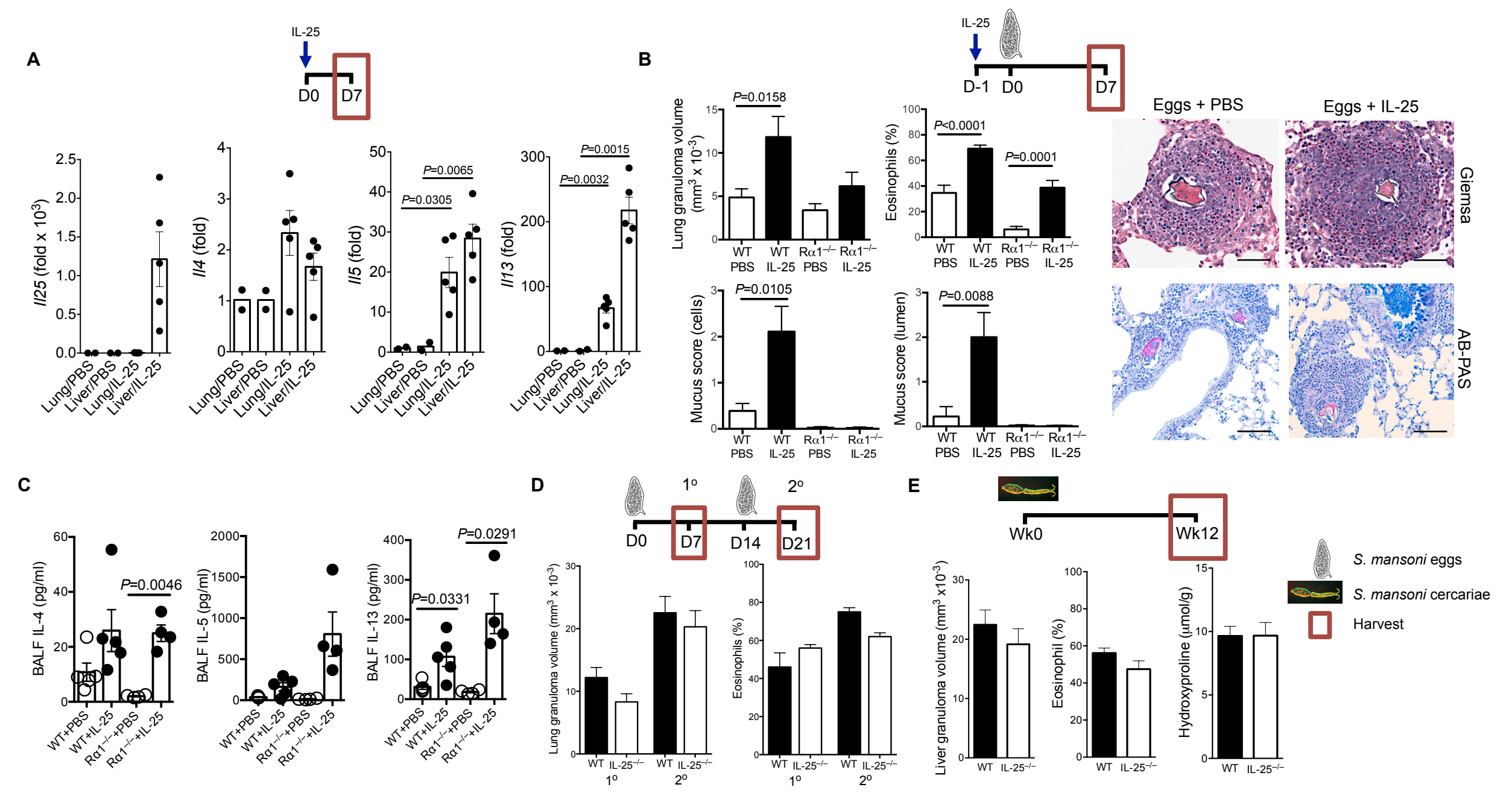




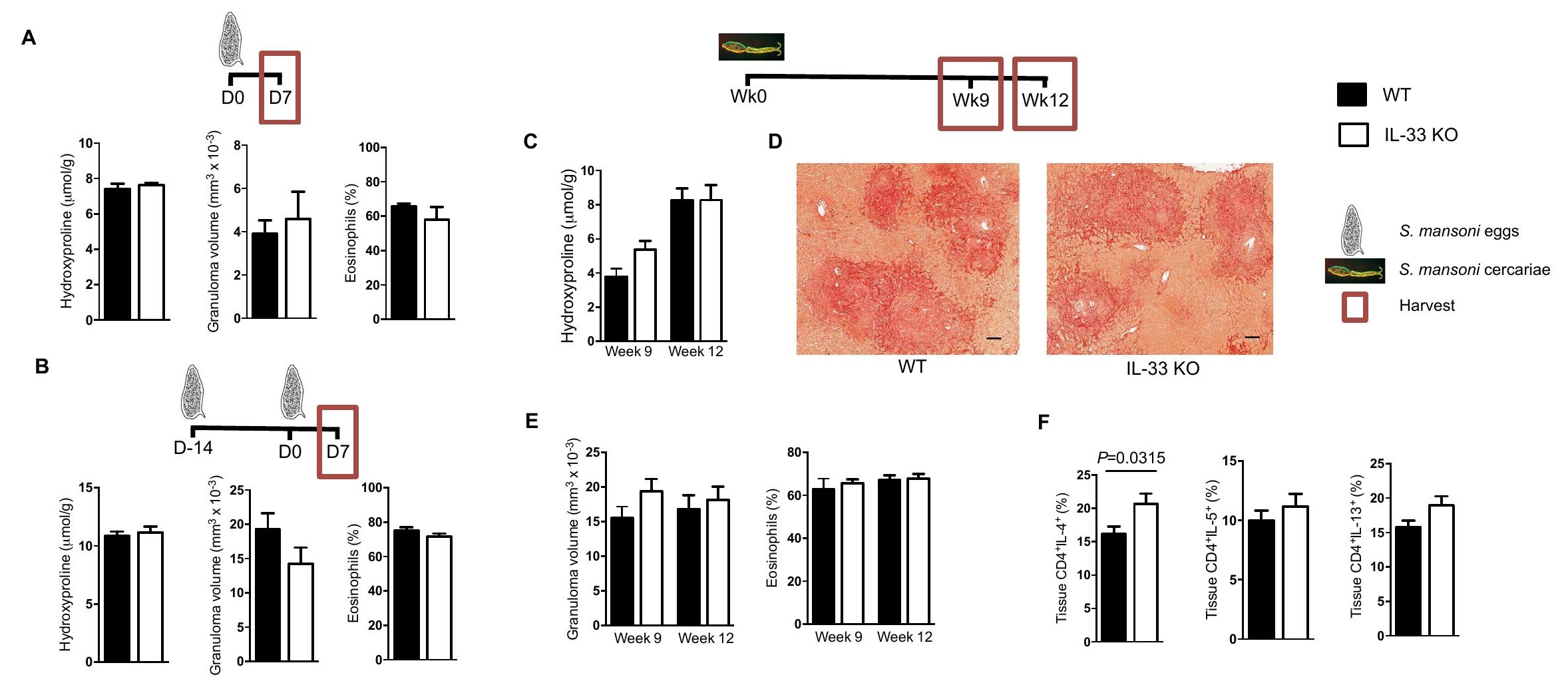


A
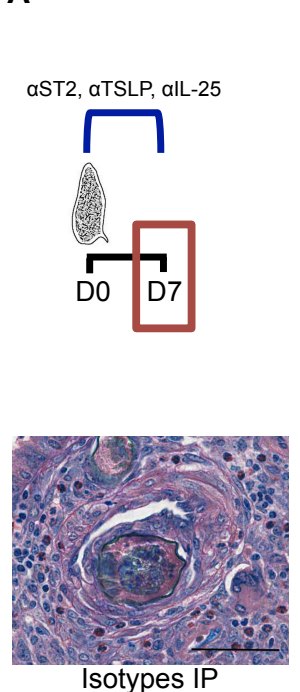

C
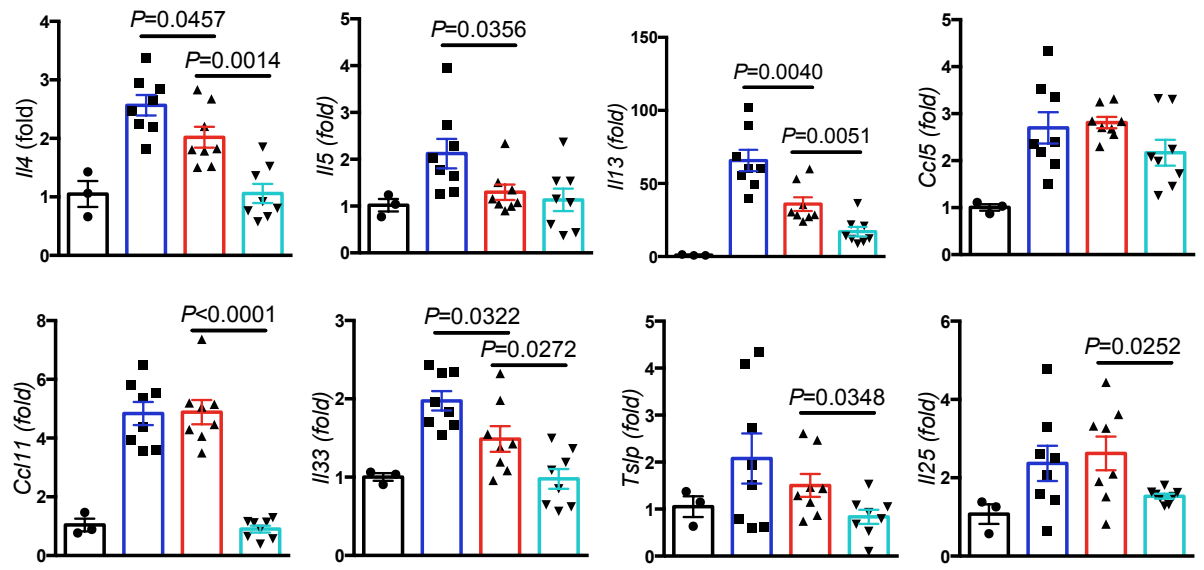

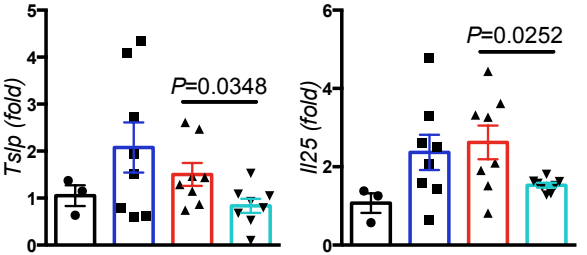

S. mansoni eggs

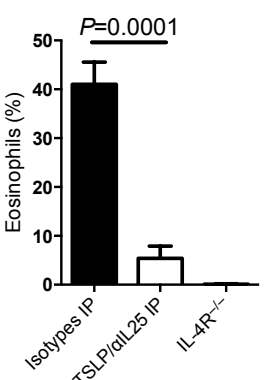

B
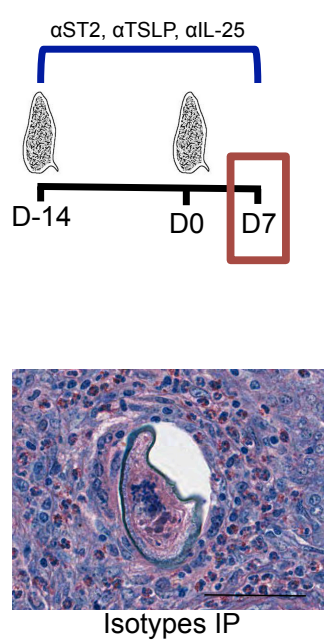

Isotypes IP
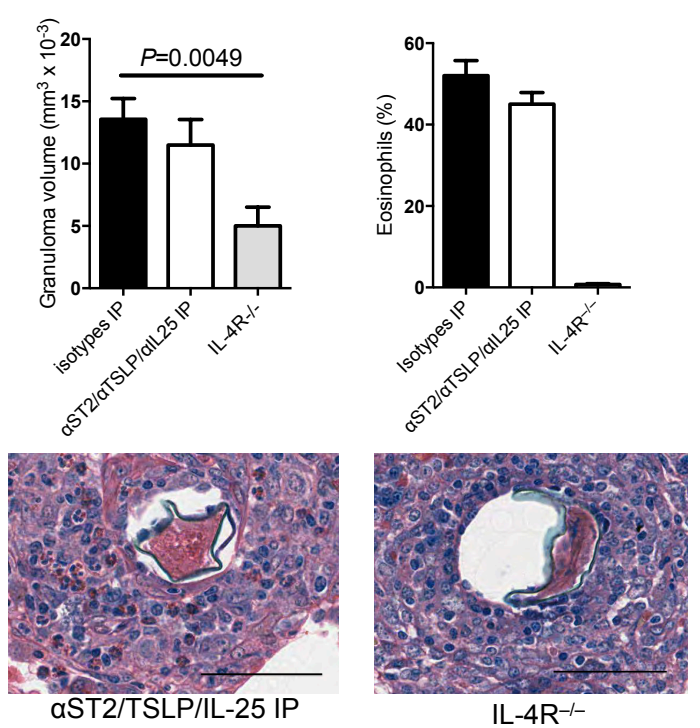

D
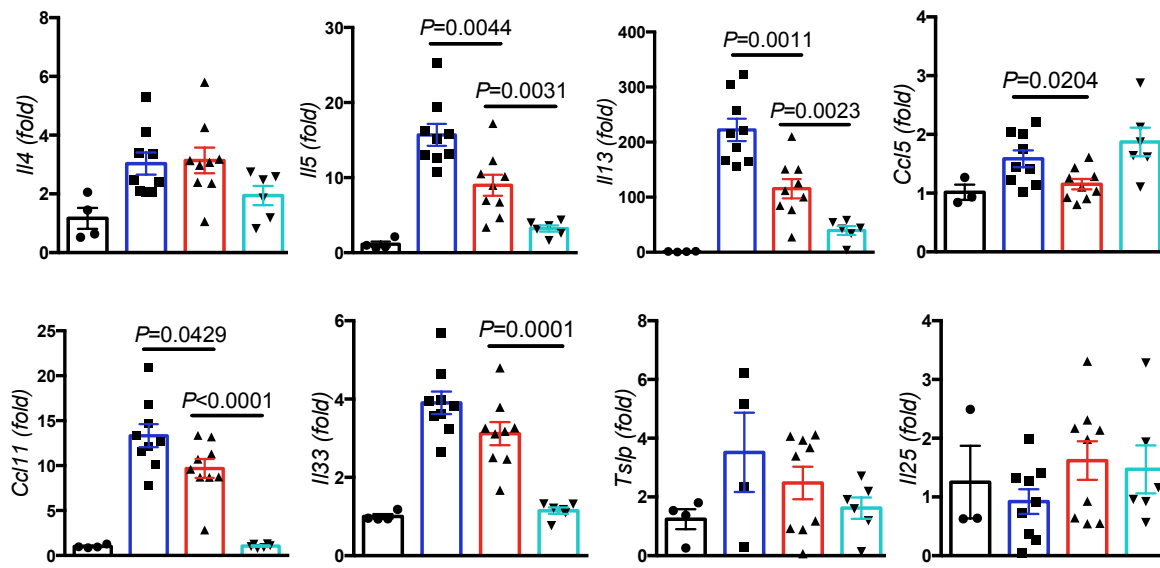

$\square$ Naive $\square$ Isotypes IP $\square$ aST2/aTSLP/alL-25E IP $\quad \square \quad$ IL-4R ${ }^{-/-}$ 


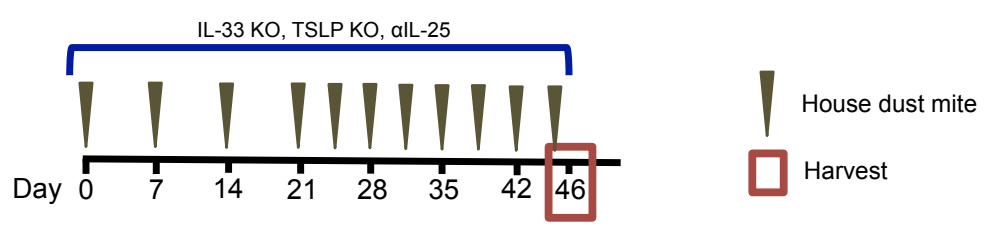

A

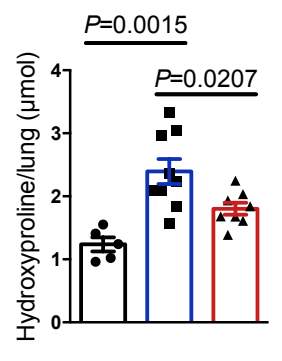

C

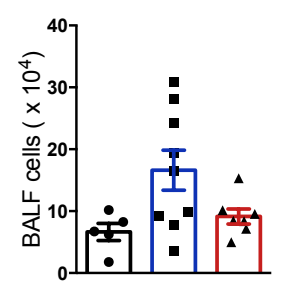

D

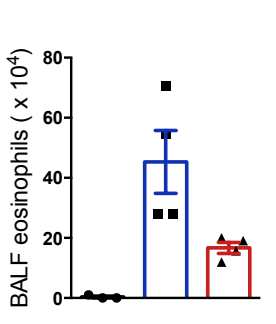

$\mathbf{F}$

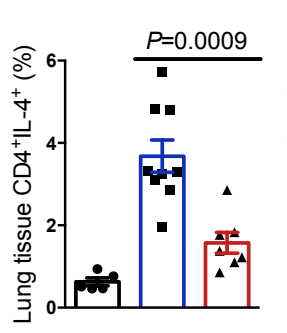

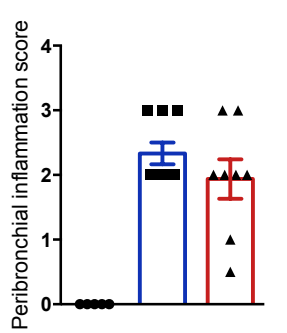
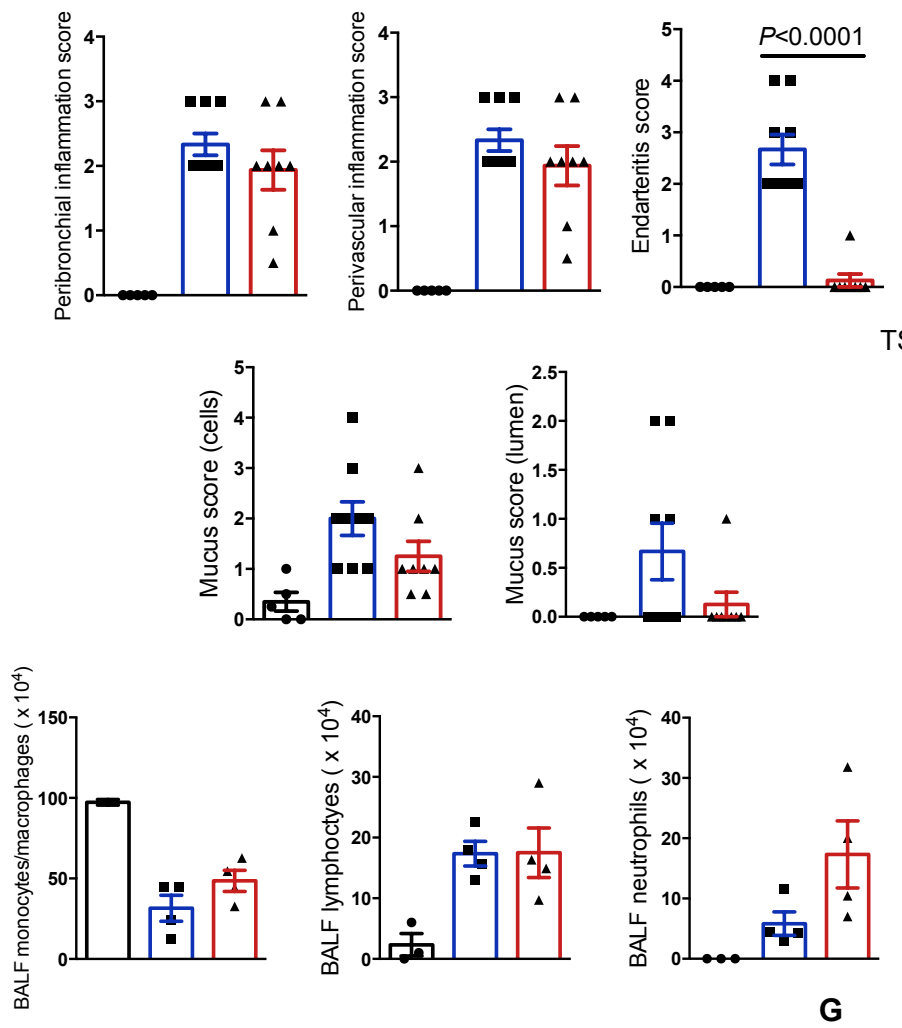

G
Istotypes IP / HDM IN

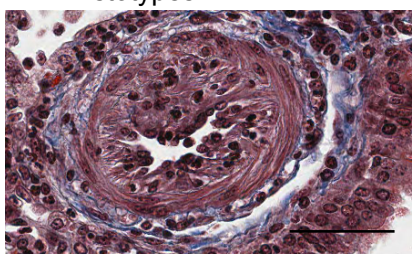

TSLP/IL-33 DKO + alL-25 IP / HDM IN

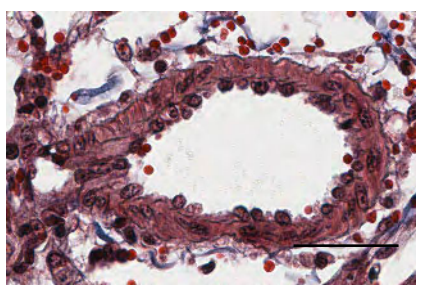

E
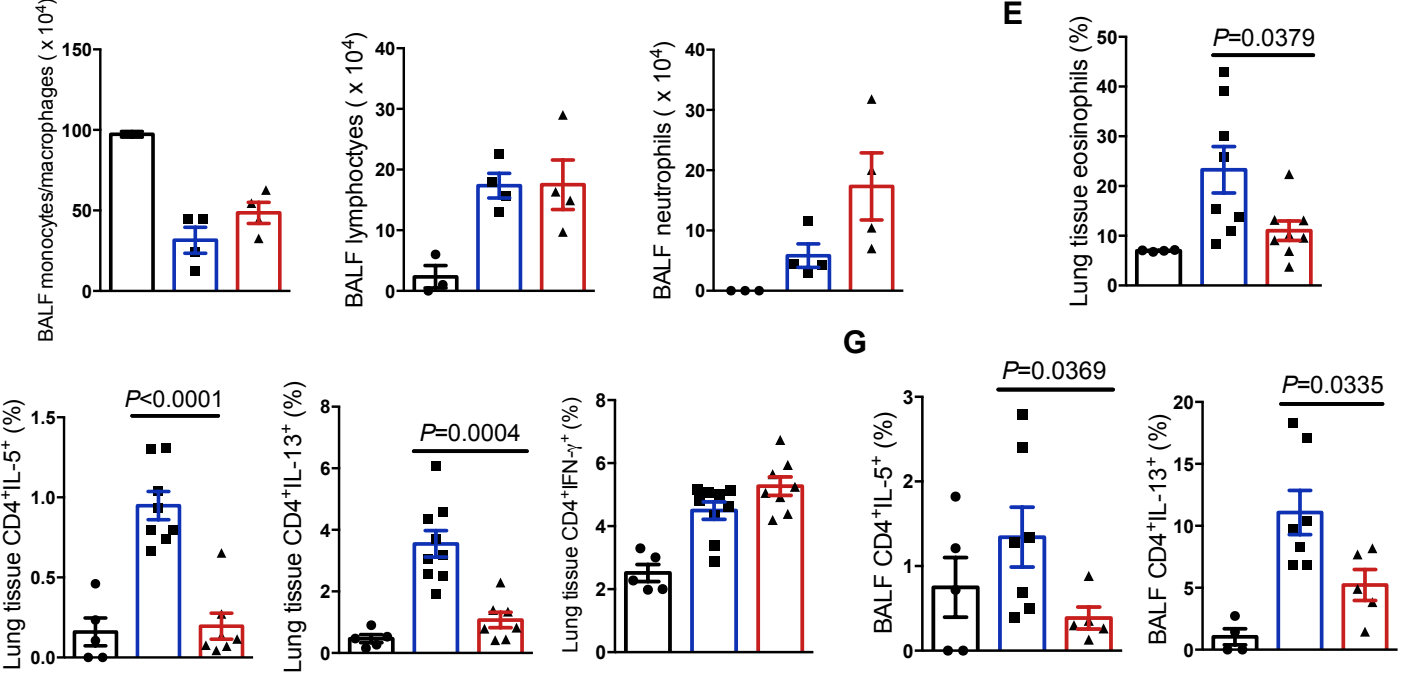


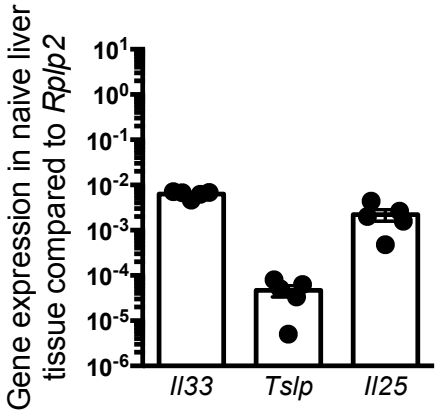


A

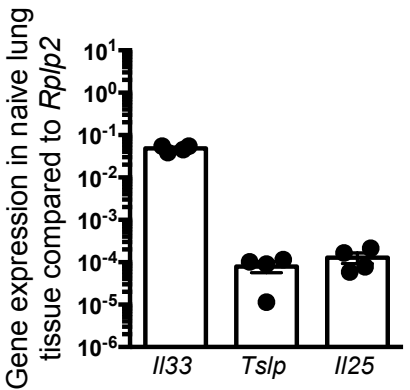

B

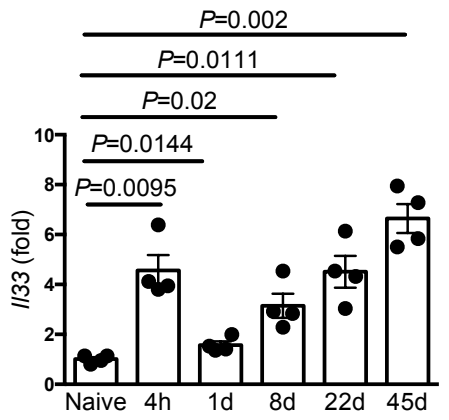

Time after initial HDM administration
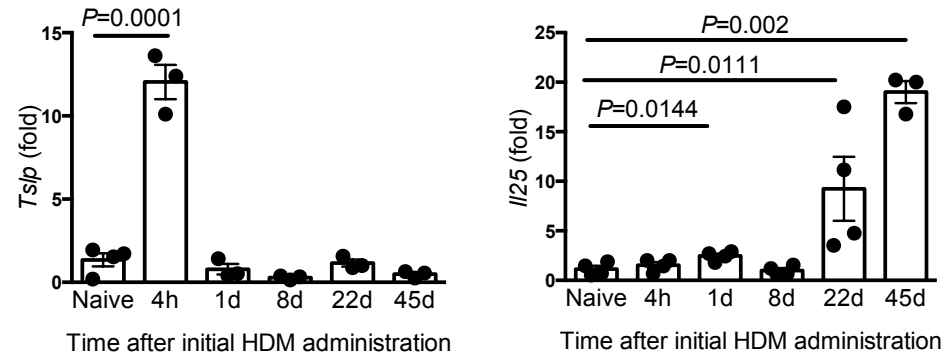
aST2, aTSLP, alL-25

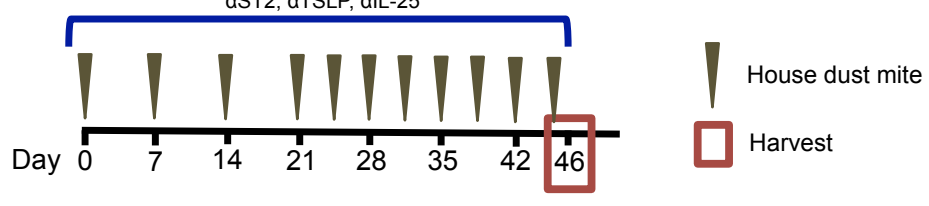

B

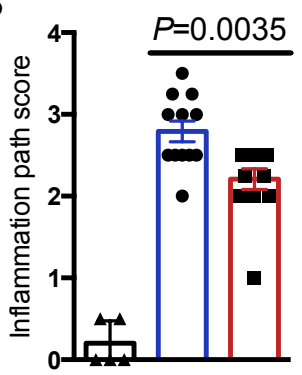

C

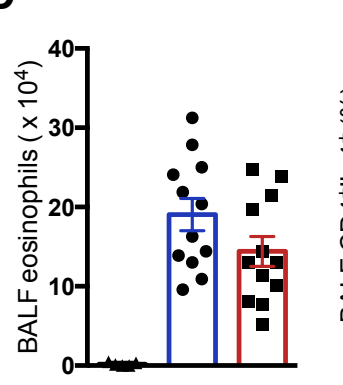

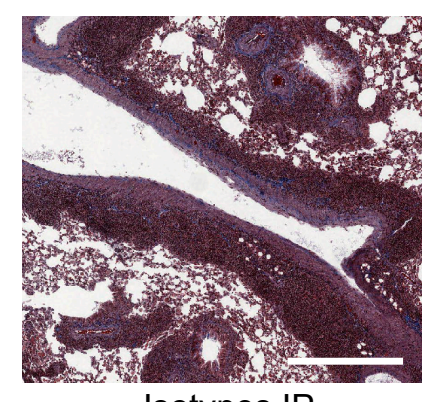

Isotypes IP
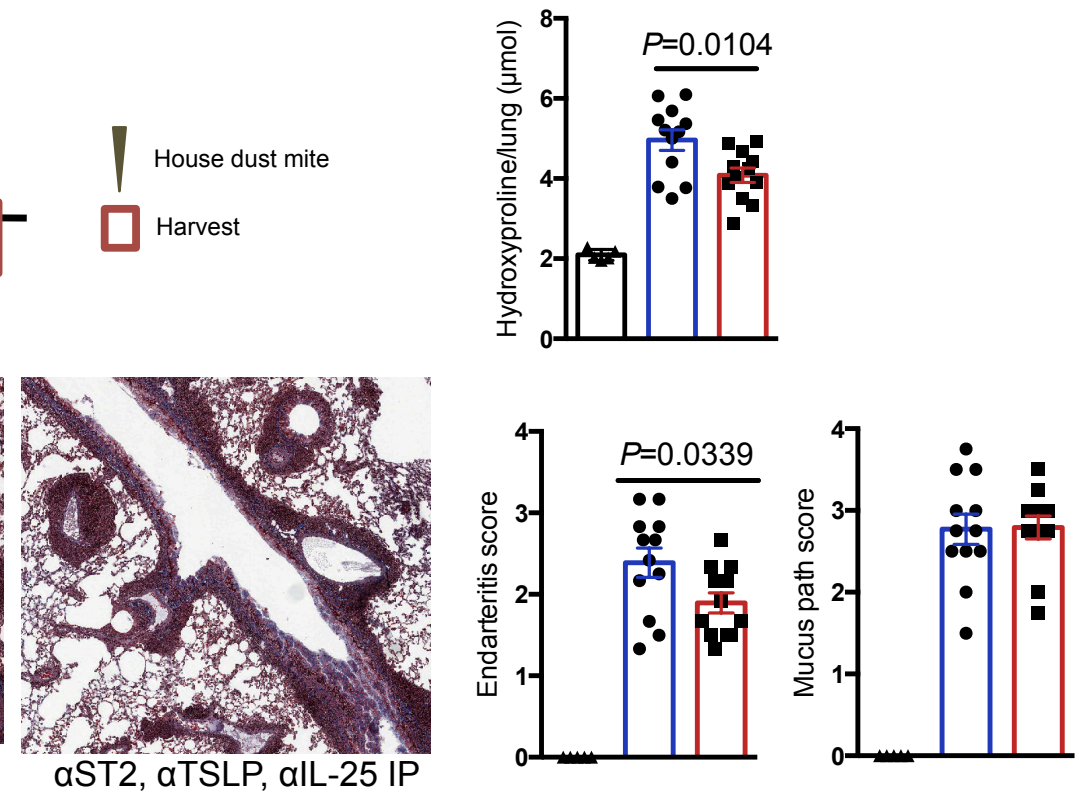

D
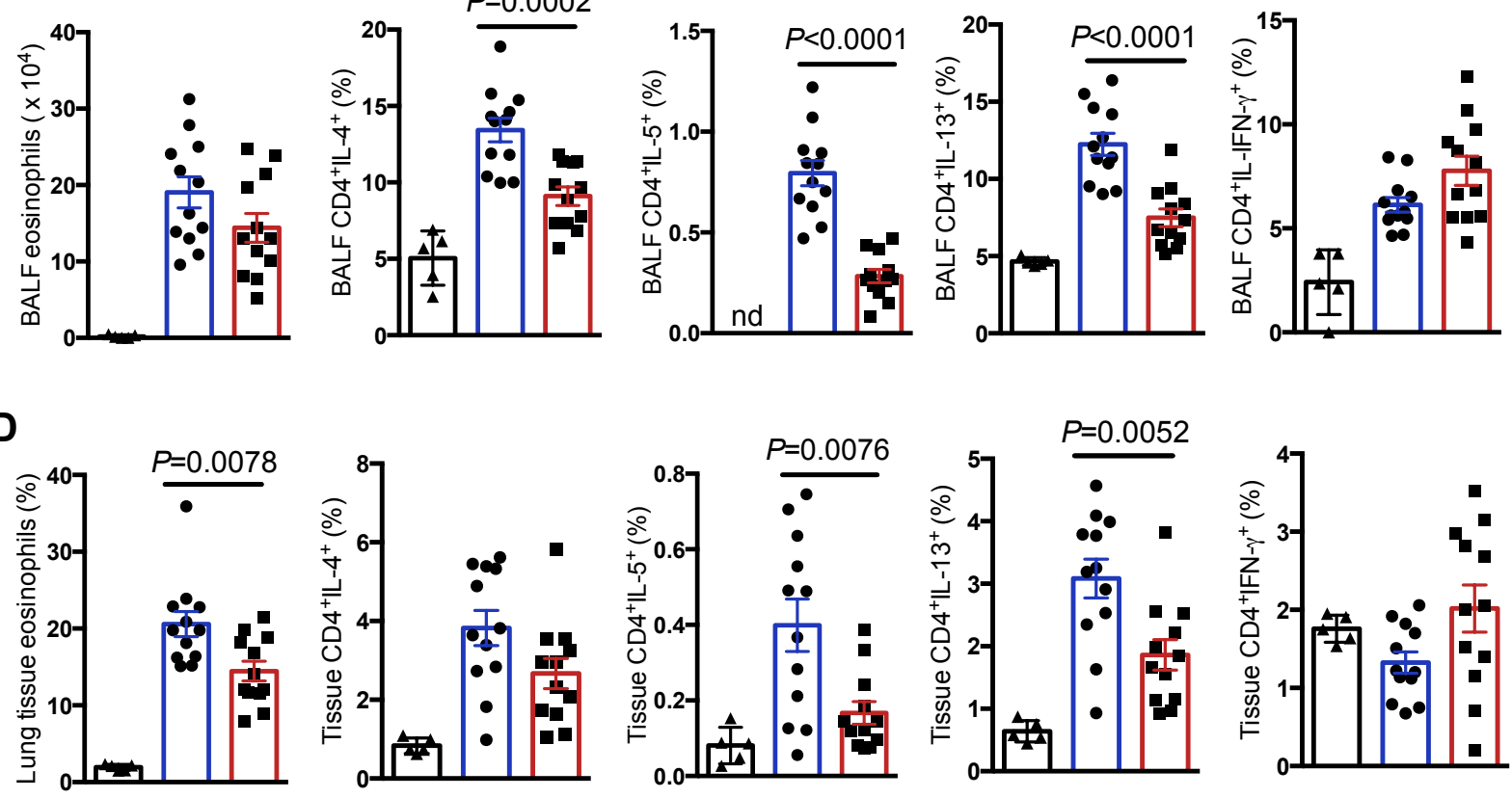

Isotypes IP / saline IN

Isotypes IP / HDM IN

aST2, aTSLP, alL-25 / HDM IN 